\title{
Research on Importance Evaluation of Complex Product Parts Based on Multilayer Complex Network
}

\author{
Weiming Yang $\mathbb{D}^{1},{ }^{1}$ Congdong $L i \mathbb{D}^{1},{ }^{1}$ Yinyun $Y u \mathbb{D}^{1},{ }^{1}$ and Mingsheng Zhong ${ }^{2}$ \\ ${ }^{1}$ School of Management, Jinan University, Guangzhou, Guangdong 510632, China \\ ${ }^{2}$ Gree Electric Appliances, Inc.of Zhuhai, Zhuhai, Guangdong 519099, China \\ Correspondence should be addressed to Congdong Li; licd@jnu.edu.cn
}

Received 26 May 2021; Revised 12 July 2021; Accepted 13 August 2021; Published 2 September 2021

Academic Editor: Jinliang Wang

Copyright $\odot 2021$ Weiming Yang et al. This is an open access article distributed under the Creative Commons Attribution License, which permits unrestricted use, distribution, and reproduction in any medium, provided the original work is properly cited.

The identification and evaluation of important parts play an important role in effective production arrangement and shortening the product development in the product design stage. In this paper, a complex product node importance evaluation method based on multilayer network is proposed to identify and evaluate importance parts faster. First, a complex product design expression network based on "function behavior structure (FBS)" multilayer complex network is established. Second, the evaluation index system of important design parts for complex products based on multilayer network is constructed. Third, a three-parameter grey relational model based on the fuzzy analytic hierarchy process and the Gini coefficient method is proposed. Finally, this method is available and feasible through taking the large permanent magnet synchronous centrifugal unit as an example.

\section{Introduction}

Complex product design process has the characteristics of diversity, nonlinearity, uncertainty, and evolution, which needs the efficient support and cooperation of various resources. Its architecture is highly complex and high dimensional. In this complex design process, on the one hand, companies have to invest a lot of manpower, material resources, and financial resources and require precise budgets and production arrangements. On the other hand, it is often interdisciplinary and accompanied by grey information. The experience and knowledge of experts in various fields are needed. Complex network can well represent the complex product design process. Many scholars use complex networks to realize the design control of complex products in recent years. Among them, the identification of important parts of complex products is of great value to improve the efficiency and sensitivity of the design $[1,2]$.

The identification of important nodes has attracted the attention of researchers and practitioners. Many diffusion models have been proposed to identify important nodes in complex networks [3-5]. The method commonly used to identify the most influential node set is the central node. Many methods can be used to measure the centrality of complex network structure, such as network degree, intermediate number, and tightness. However, measuring the centrality of the network structure to identify the nodes with maximum influence is not the best way to solve this problem. There are few studies on the evaluation of important nodes related to complex product engineering design. However, the research on the important nodes of complex products is of great significance for the design knowledge of control nodes. Therefore, this paper proposes a new important node identification method based on the multilayer network. Firstly, a multilevel design expression network considering the function, structure, and behavior of a complex product design is constructed. Then, a comprehensive new evaluation index system about network characteristics and component value is established. In addition, an improved threeparameter interval grey number model is used for evaluation considering the multidomain heterogeneity and difficult acquisition of knowledge. In the improvement, in order to better adapt to the research of this paper, we integrate the fuzzy analytic hierarchy process and the Gini coefficient method to improve the method. 
The remainder of this paper is organized as follows. After reviewing some relevant literature in Section 2, we describe the research problem in Section 3 and give determination of evaluation index system in Section 4. In Section 5, we give the research methodology in this paper. In Section 6, we provide a case study about large permanent magnet synchronous centrifugal unit. Section 7 concludes this work.

\section{Literature Review}

Many scholars have proposed many methods for important node identification in recent years. Lu et al. [6] designed a cascade discount algorithm to solve the influence maximization problem. Gong et al. [7] proposed a discrete particle swarm optimization algorithm to optimize the local influence criterion. The representations and update rules for the particles are redefined in the proposed algorithm. Bao et al. [8] proposed a heuristic clustering ( $\mathrm{HC}$ ) algorithm based on the similarity index to classify nodes into different clusters, and finally, the center nodes in clusters are chosen as the multiple spreaders. HC algorithm not only ensures that the multiple spreaders are dispersively distributed in networks but also avoids the selected nodes to be very "negligible." Cui et al. [9] proposed a degree-descending search strategy (DDS) and developed an evolutionary algorithm that is capable of improving the efficiency significantly by eliminating the time-consuming simulations of the greedy algorithms. Li et al. [10] proposed an improved label propagation algorithm named LPA-MNI by combining the modularity function and node importance with the original LPA. Berberler et al. [11] conducted node importance analysis in wheel-related networks by a method of evaluating node importance by node contraction based on network agglomeration in communication networks. Sun et al. [12] proposed an entropy-based self-adaptive node importance evaluation method to evaluate node importance objectively.

Some of the above studies are aimed at pure networks, and most of them only study some characteristics of networks. It is difficult to reflect the importance of nodes in an all-round way and cannot effectively identify the important nodes in practical application. We start with multilayer network and establish a new index. This index system includes the value of parts in the design, which is more comprehensive.

There are many research studies on the description network of complex products, mainly focusing on the research of single-layer and weighted complex networks. Bencherif and Mouss [13] proposed a product development process model in an innovation context and strategy framework of design process and project management. The process modelling is based on complex network theory, to improve characterization analysis for product development process modelling. Li et al. [14] focused on the impacts of organization-component executing patterns on the risk propagation in CPD interdependent networks considering the limited risk resisting capacity of organizations. Li et al. [15] developed a general model based on complex network to depict the dynamic design change propagation. $\mathrm{Nu}-$ merical simulations are conducted to explore the general law of the propagation and investigate the influences of design change tolerance capacity distribution (alpha, beta), attack strategies, and recovery capacity (gamma). Li et al. [16], for the first time, applied the SoV into the research on quality risk propagation of complex product collaborative manufacturing supply chain network. Yin et al. [17] proposed a method for identifying the influential parts of a CMP based on complex network theory from the perspective of reliability. It is used to identify the influential parts in each community of products. Yu et al. [18] built a directed weighted complex product network model to represent the product structure under given requirement by defining interconnections among parts. Zhang et al. [19] proposed a novel HEO modelling method based on complex networks' theory. Zhang et al. [20] proposed a new four-phase routing approach based on weighted and directed complex networks for multisource design change propagation.

It can be seen from the existing design networks that single-layer networks are often used to study their properties. However, some design factors will be ignored. So, this paper uses a comprehensive design process description model, FBS model, to describe the design process. The comprehensive evaluation index system is established. Then, the three-parameter interval grey number grey relational model is used to evaluate the important nodes.

There are many studies on three-parameter interval grey number relational model. Considering the value information of the three-parameter interval grey number and the risk attitude of decision maker, Zhang et al. [21] advanced a multiattribute group grey target decision-making method based on a three-parameter interval grey number. From the perspective of three-way decision space, Li and Zhang [22] combined the theories of interval concept lattice and threeway decision and then put forward interval three-way decision space theory. To solve decision-making problems having the characteristics of grey systems, Li et al. [23] introduced the three-parameter interval grey number to measure the evaluation index. Chen and Chen [24] proposed a dynamic multiattribute decision-making method based on the prospect theory for dealing with the dynamic multiattribute decision-making problem with the three-parameter interval grey number. Aiming at the multiple attribute decision-making problem with three-parameter interval grey numbers, Li and Zhang [25] proposed a grey-incidence clustering decision-making method based on regret theory. In consideration of the fuzziness and the uncertainty in decision information, $\mathrm{Li}$ et al. [26] proposed a three-parameter interval grey linguistic variable decision-making method based on projection model and prospect theory. It can be seen from the previous literature that scholars have carried out a lot of research studies on three-parameter interval grey number relational model. However, there are still some areas to be studied about the determination of its weight. In order to make it more suitable for this paper, this study comprehensively considers its weight setting.

To sum up, we can see that the single-layer network is mostly used to analyze the network center from the expression of the design process. The research on the identification method of important network nodes mainly adopts 
the search algorithm. In the process of subcomplex product design, the identification of important nodes is very important, which determines the speed and quality of the following links. Firstly, this paper establishes a multilayer network model considering the functional behavior structure knowledge in the process of the product design. Then, a new evaluation index is determined for the network model, including the network characteristics and the value of parts. We improve the three-parameter interval grey number relational model to adapt to the research. Finally, we use the three-parameter grey relational model based on TFAHP and Gini coefficient method to evaluate and rank the important nodes in engineering change. The research framework is shown in Figure 1.

\section{Problem Description}

Previous studies have shown that the FBS model is a more comprehensive description model of the design process, in which the function represents what the design object is used to do, behavior describes what the design object does, and structure specifies what the design object is. In the process of complex product design, the FBS model can be used to formalize the parameters of the parts according to the new function, behavior, and structure requirements. In addition, Hamraz et al. also emphasized that the use of the FBS model to express the relationships in complex products can effectively improve the product design. Therefore, this paper considers the FBS relationship to formalize the complex product design process.

The realization of function is based on the exchange of material, information, and energy between the product and the external environment. When two parts share the functions in the form of material flow, information flow, and energy flow, we define that there is a functional relationship between the parts. Product function is an abstract concept, which can make the function relationship have strong concealment and often cannot be perceptual cognition by designers.

Behavior is a bridge between the function and the structure and an objective description of function realization. It contains three independent implicit behavior attributes: mechanical (strength, inertia, elasticity, etc.), electrical characteristics (conduction, resistance, charging, etc.), and thermal effects (conduction, temperature change, absorption, etc.). Due to the behavior requirements of products, the combination of behavior attributes can be formed between parts with the same behavior attributes.

Structure is the physical carrier of function, which emphasizes the specific composition of the physical entity to realize the function. In particular, structural attributes are explicit attributes used to describe physical entities, which mainly include geometry (size, shape, etc.), material (type, volume, density, etc.), surface (surface roughness, texture, etc.), and controller (microchip, relay, etc.). Similarly, due to the structural requirements of products, the combination of structural attributes exists between components with the same structural attributes (Figure 2). The multilayer network construction process is shown in Figure 2.
The $n$ components of a complex product and their associated relationships are represented as a single-layer subnetwork $G \alpha=(V, E \alpha, W \alpha)$, where $\alpha=1,2,3$ are the network levels. It represents the network level and represents the single-layer subnetwork type (functional network, behavioral network, and structural network). $V=(v i \mid i=1,2, \ldots, N)$ is the set of network nodes. It represents the components set. $E \alpha=\{e i \alpha, j \mid i=1,2, \ldots, N$; $j=1,2, \ldots, N ; i \neq j\}$ is the set of connected edges of the network. It represents the association set. $W \alpha=$ $\{w i \alpha, j \mid i=1,2, \ldots, N ; j=1,2, \ldots, N ; i \neq j\}$ is the weight set of connected edges. It represents the association strength set. For node $v i$ and node $v j$ in the network, if there is an association relationship between the corresponding components, then there is a connected edge ei $\alpha, j=1$.

\section{Determination of Evaluation Index System}

Compared with the traditional complex network, the multinetwork model can better reflect the diversity of association relations in complex products and the rich topology characteristics in complex products. In order to evaluate the influence of each node, we need to comprehensively consider the local attributes, global attributes, and their specific significance in complex products from multiple perspectives. At the same time, we consider the indicators that can reflect the properties of parts of complex products to form a comprehensive evaluation index. The detailed indicators are as follows:

(1) Clustering coefficient: it refers to the parameters used to measure the clustering of network nodes. Intuitively speaking, clustering coefficient describes the possibility that a node's friends are still friends. The higher the clustering coefficient of a node, the more likely it is that its friend is still a friend, and the more important it is in the whole network circle. Generally, assuming that a node $i$ in an undirected network has $k$ adjacent nodes, then the ratio of the actual number of edges to the possible number of edges among the $k$ nodes is called the clustering coefficient: $C_{i}=\left(E_{i} /(k(k-1) / 2)\right) * k$, where $k$ is the degree of node $i$, which is equivalent to counting the number of friends of node $i$ and $E_{i}$ is the actual number of edges between $k$ nodes connected with node $i$.Suppose there are $n$ nodes in a complex network; then, the Erdos number of each node is defined as follows: $D_{i}=(1 /(N-1)) \sum_{i, j \neq i} d_{i j}$, where $d_{i j}$ is the length of the shortest path from node $i$ to node $j$.

(2) Erdos: assuming that the current node is Erdos and the Erdos number of other nodes is the length of the shortest path from the current node to other nodes, then the Erdos number of the current node is the length of the average path from the current node to other nodes.

(3) Node betweenness: the number of paths that all shortest paths in the network pass through this node. The betweenness $B_{i}$ of vertex $i$ is defined as 


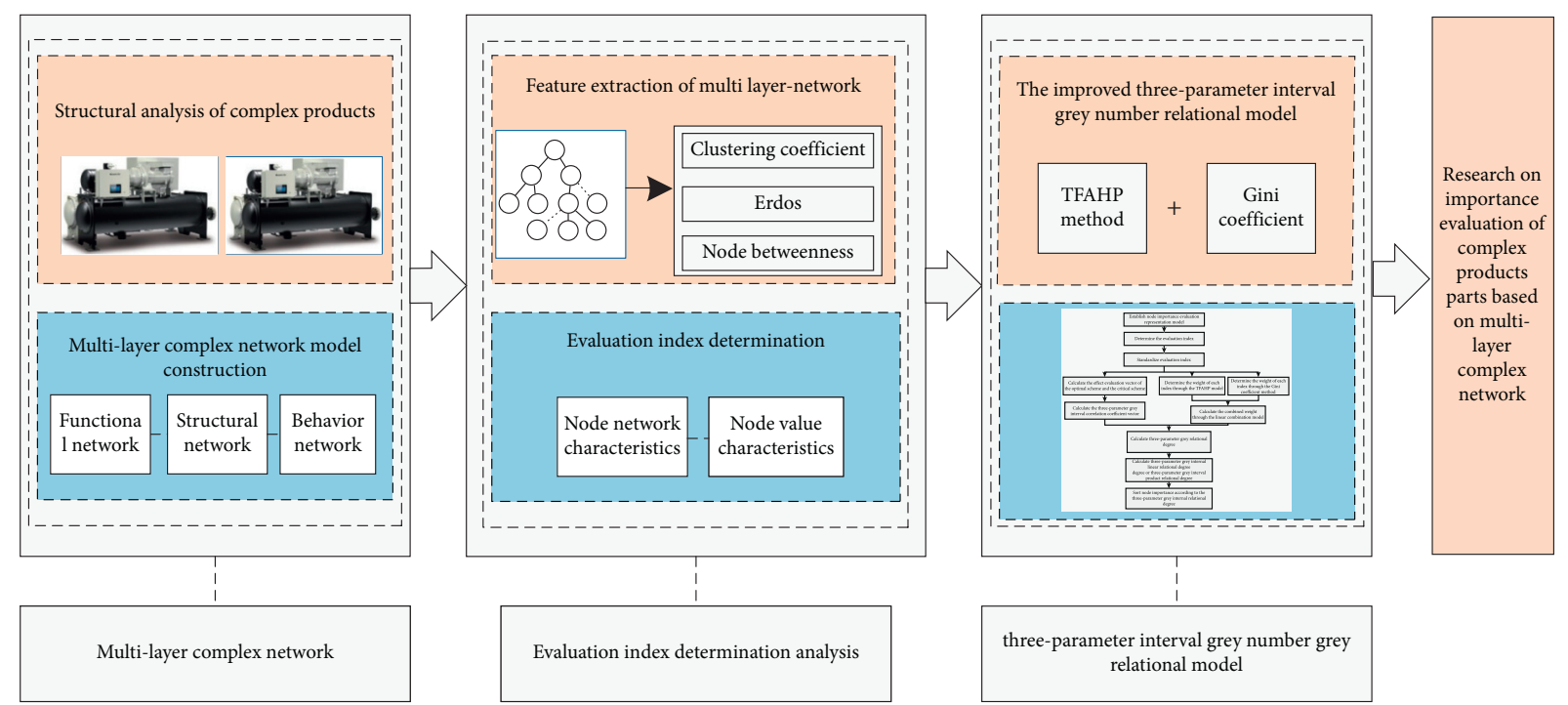

FIGURE 1: Framework of the proposed complex product node importance evaluation method.
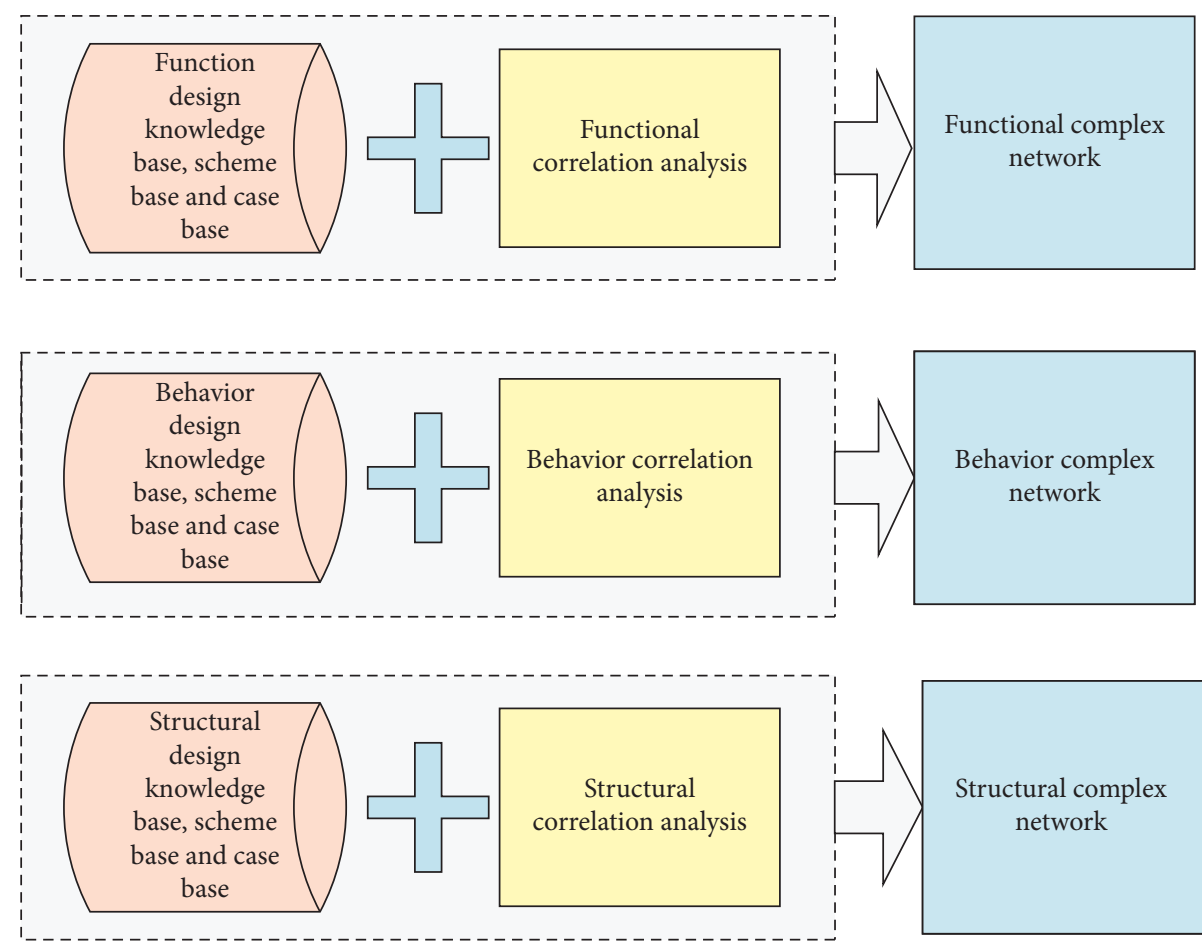

FIgURE 2: Multilayer complex network analysis of complex product design knowledge.

$B_{i}=\sum_{j, k \in V}\left(n_{j k}(i) / n_{j k}\right)$, where $n_{j k}(i)$ is the number of shortest paths between nodes $j$ and $k$.

(4) Parts value: the engineering change of different parts needs different change costs. And, different nodes have different values. Therefore, the node's own attribute is the value of node which is used as the evaluation index of node importance. It can be expressed as $C_{\left(v_{i}\right)}=c_{\left(v_{m}\right)}+c_{\left(v_{l}\right)}+c_{\left(v_{e}\right)}+c_{\left(v_{o}\right)}$, where $c_{i}$ is the total value of node $i$ and $C_{\left(v_{m}\right)}$ is the material cost if node $i$. It refers to the cost of product standard consumption, supporting raw materials, product accessories, and various materials used for production or providing services. It mainly includes the purchase price, related taxes, freight, loading and unloading fees, insurance premiums, and other costs that can be directly attributable to the acquisition of materials. $C_{\left(v_{i}\right)}$ is the labor cost. It refers to the remuneration and other expenses paid to employees in order to obtain the services provided by employees. It mainly includes the salary, bonus, allowance, 
welfare, and education fund. $C_{\left(v_{m}\right)}$ is the manufacturing cost. It refers to energy consumption, manufacturing accessories, labor insurance, and office and fixed expenses. $C_{\left(v_{0}\right)}$ is the other costs, some consumption including fuel cost, power cost, office cost, and depreciation consumed by each production unit.

\section{Research Methodology}

5.1. Three-Parameter Interval Grey Number. From the definition of three-parameter interval grey number, it can be known that it refers to the interval grey number where the center of gravity point with the greatest possible value is known. It can be marked as $A(\otimes)=[\underline{a}, \tilde{a}, \bar{a}]$, where $\underline{a} \leq \tilde{a} \leq \bar{a}$, and $\underline{a}$ and $\bar{a}$ are the upper and lower limits of the interval, respectively. $\widetilde{a}$ is called the "center of gravity" point $([27,28])$.

When two of the three parameters $\underline{a}, \tilde{a}$, and $\bar{a}$ are the same, the three-parameter interval grey number degenerates to the interval grey number. When $\underline{a}=\tilde{a}=\bar{a}$, the threeparameter interval grey number degenerates to the real number. In fact, the interval grey number and the real number are special cases of the three-parameter interval grey number.

Its algorithm is similar to the interval grey number. Let three-parameter interval grey number be $A(\otimes)=[\underline{a}, \tilde{a}, \bar{a}]$ and $B(\otimes)=[\underline{b}, \widetilde{b}, \bar{b}]$; then,

$$
\begin{aligned}
\text { (i) } A(\otimes)+B(\otimes)=\lfloor\underline{a}+\underline{b}, \tilde{a}+\widetilde{b}, \bar{a}+\bar{b}\rfloor \\
\text { (ii) }(A(\otimes) / B(\otimes)) \in[\min \{(\underline{a} / \underline{b}),(\underline{a} / \bar{b}),(\bar{a} / \underline{b}),(\bar{a} / \bar{b})\}, \\
\quad(\tilde{a} / \widetilde{b}), \max \{(\underline{a} / \underline{b}),(\underline{a} / \bar{b}),(\bar{a} / \underline{b}),(\bar{a} / \bar{b})\}]
\end{aligned}
$$$$
\text { (iii) } \lambda A(\otimes)=[\underline{\lambda a}, \lambda \widetilde{a}, \lambda \bar{a}], \lambda>0
$$

\subsection{Three-Parameter Interval Grey Number Grey Relational} Model. Suppose that there are $n$ alternative engineering change schemes. They constituted by evaluation schemes' set $A=\left\{a_{1}, a_{2}, \ldots, a_{n}\right\}$. The index set $S=\left\{s_{1}, s_{2}, \ldots, s_{m}\right\}$ is composed of $m$ attributes. The index value of scheme $a_{i}$ under the evaluation index $s_{j}$ can be expressed as $u_{i j}(\otimes)=$ $\left\lfloor\underline{u}_{i j}, \tilde{u}_{i j}, \quad \bar{u}_{i j}\right\rfloor\left(\underline{u}_{i j} \leq \tilde{u}_{i j} \leq \bar{u}_{i j}, i=1,2, \ldots, n ; j=1,2, \ldots, m\right)$. The effect evaluation vector of each scheme is $u_{i}(\otimes)=\left(u_{i 1}(\otimes), u_{i 2}(\otimes), \ldots, u_{i m}(\otimes)\right), i=1,2, \ldots, n$. The weight of index under each scheme is $w_{i 1}, w_{i 2}, \ldots, w_{i m}$, and $\sum_{j=1}^{m} w_{i j}=1(i=1,2, \ldots, n)$. There are different attribute indexes with different dimensions and measurement standards. In order to increase the comparability of alternatives, it is necessary to normalize the effect evaluation vector of decision alternatives. In this paper, we use the range transformation method to normalize the decision matrix.
For profitable attribute values,

$$
\begin{gathered}
\underline{x}_{i j}=\frac{\underline{u}_{i j}-\underline{u}_{j}^{\nabla}}{\bar{u}_{j}^{*}-\underline{u}_{j}^{\nabla}}, \\
\tilde{x}_{i j}=\frac{\widetilde{u}_{i j}-\underline{u}_{j}^{\nabla}}{\bar{u}_{j}^{*}-\underline{u}_{j}^{\nabla}}, \\
\bar{x}_{i j}=\frac{\bar{u}_{i j}-\underline{u}_{j}^{\nabla}}{\bar{u}_{j}^{*}-\underline{u}_{j}^{\nabla}} .
\end{gathered}
$$

For cost attribute values,

$$
\begin{gathered}
\underline{x}_{i j}=\frac{\bar{u}_{j}^{*}-\bar{u}_{i j}}{\bar{u}_{j}^{*}-\underline{u}_{j}^{\nabla},} \\
\tilde{x}_{i j}=\frac{\bar{u}_{j}^{*}-\tilde{u}_{i j}}{\bar{u}_{j}^{*}-\underline{u}_{j}^{\nabla}} \\
\bar{x}_{i j}=\frac{\bar{u}_{j}^{*}-\bar{u}_{i j}}{\bar{u}_{j}^{*}-\underline{u}_{j}^{\nabla}},
\end{gathered}
$$

where $\bar{u}_{j}^{*}=\max _{1 \leq i \leq n}\left\{\bar{u}_{i j}\right\}, \underline{u}_{j}^{\nabla} \min _{1 \leq i \leq n}\left\{\underline{u}_{i j}\right\}, j=1,2, \ldots, m$.

Let the normalized effect evaluation vector be

$$
x_{i}(\otimes)=\left(x_{i 1}(\otimes), x_{i 2}(\otimes), \ldots, x_{i m}(\otimes)\right), \quad i=1,2, \ldots, n,
$$

where $x_{i j}(\otimes) \in\left[\underline{x}_{i j}, \tilde{x}_{i j}, \bar{x}_{i j}\right]$ is a three-parameter interval grey number in $[0,1]$.

It is recorded that $\underline{x}_{j}^{+}=\max _{1 \leq i \leq n}\left\{\underline{x}_{i j}\right\}, \quad \tilde{x}_{j}^{+}=$ $\max _{1 \leq i \leq n}\left\{\tilde{x}_{i j}\right\}, \quad \bar{x}_{j}^{+}=\max _{1 \leq i \leq n}\left\{\underline{x}_{i j}\right\}, \quad \underline{x}_{j}^{-}=\min _{1 \leq i \leq n}\left\{\underline{x}_{i j}\right\}$, $\tilde{x}_{j}^{-}=\min _{1 \leq i \leq n}\left\{\widetilde{x}_{i j}\right\}$, and $\bar{x}_{j}^{-}=\min _{1 \leq i \leq n}\left\{\bar{x}_{i j}\right\}(j=1,2$, $\ldots, m)$. Then, the $m$-dimensional three-parameter nonnegative interval grey number vectors,

$$
\begin{aligned}
& x^{+}(\otimes)=\left\{x_{1}^{+}(\otimes), x_{2}^{+}(\otimes), \ldots, x_{m}^{+}(\otimes)\right\}, \\
& x^{-}(\otimes)=\left\{x_{1}^{-}(\otimes), x_{2}^{-}(\otimes), \ldots, x_{m}^{-}(\otimes)\right\},
\end{aligned}
$$

are called ideal optimal scheme effect evaluation vectors and critical scheme effect evaluation vectors, respectively [29].

We assume that the grey interval relational degree of the normalized effect evaluation vector $x_{i}(\otimes)$ of scheme $A_{i}$ with respect to the ideal optimal scheme effect evaluation vector $x^{+}(\otimes)$ is $G\left(x^{+}(\otimes), x_{i}(\otimes)\right)$. And, the grey interval relational degree of the critical scheme effect evaluation vector $x^{-}(\otimes)$ is $G\left(x^{-}(\otimes), x_{i}(\otimes)\right)$. Assume that the weights of two grey relational degrees are $\alpha_{1}, \alpha_{2}\left(\alpha_{1}+\alpha_{2}=1\right)$. Then,

$$
G\left(x_{i}(\otimes)\right)=\alpha_{1} G\left(x^{+}(\otimes), x_{i}(\otimes)\right)+\alpha_{2}\left[1-G\left(x^{-}(\otimes), x_{i}(\otimes)\right)\right], \quad i=1,2, \ldots, n,
$$


is the three-parameter grey interval linear relational degree of the effect evaluation vector $x_{i}(\otimes)$.

$$
G\left(x_{i}(\otimes)\right)=\left[G\left(x^{+}(\otimes), x_{i}(\otimes)\right)\right]^{\alpha_{1}}+\left[1-G\left(x^{-}(\otimes), x_{i}(\otimes)\right)\right]^{\alpha_{2}}, \quad i=1,2, \ldots, n
$$

is the three-parameter grey interval product relational degree of the effect evaluation vector $x_{i}(\otimes)$ (according to [28] and experts' opinion, this paper is set to $\lambda=0.5, \xi=0.5$, and $\left.\beta_{1}=\beta_{2}=0.5\right)$.

The distribution probability of barycenter point with the highest probability of taking the value of three-parameter interval grey number $x_{i j}(\otimes) \in\left[\underline{x}_{i j}, \tilde{x}_{i j}, \bar{x}_{i j}\right]$ is $f\left(\tilde{x}_{i j}\right) \geq \sigma$. Normally, $\sigma \geq 60 \%$. If $\sigma \leq 60 \%$, it indicates that the decision is wrong, and the most likely value needs to be determined again. Based on the center of gravity, we can build a threeparameter interval grey number relational degree evaluation model.

Definition 3. For the three-parameter interval grey number $x_{i j}(\otimes) \in\left[\underline{x}_{i j}, \tilde{x}_{i j}, \bar{x}_{i j}\right]$,

$$
\gamma_{i j}^{+}=\frac{3}{5} \times \frac{\tilde{m}^{+}+\eta \tilde{M}^{+}}{\widetilde{\Delta}_{i j}^{+}+\eta \tilde{M}^{+}}+\frac{2}{5}\left[(1-\beta) \frac{\underline{m}^{+}+\eta \underline{M}^{+}}{\underline{\Delta}_{i j}^{+}+\eta \underline{\underline{M}}} \beta \frac{\bar{m}^{+}+\eta \bar{M}^{+}}{\bar{\Delta}_{i j}^{+}+\eta \bar{M}^{+}}\right],
$$

is called the three-parameter grey interval relational coefficient of subfactor $x_{i j}$ with respect to ideal factor $x_{j}^{+}$. $\eta \in(0,1)$ is the resolution coefficient. $\beta \in(0,1)$ is the decision preference coefficient (according to [28] and experts' opinion, normally, $\eta=\beta=0.5$ ), where

$$
\begin{aligned}
& \underline{\Delta}_{i j}^{+}=\left|\underline{x}_{j}^{+}-\underline{x}_{i j}\right|, \\
& \tilde{\Delta}_{i j}^{+}=\left|\tilde{x}_{j}^{+}-\tilde{x}_{i j}\right|, \quad i=1,2, \ldots, n ; j=1,2, \ldots, m, \\
& \bar{\Delta}_{i j}^{+}=\left|\bar{x}_{j}^{+}-\bar{x}_{i j}\right|, \\
& \underline{m}^{+}=\min _{1 \leq i \leq n} \min _{1 \leq j \leq m} \underline{\Delta}_{i j}^{+}, \\
& \tilde{m}^{+}=\min _{1 \leq i \leq n} \min _{1 \leq j \leq m} \tilde{\Delta}_{i j}^{+}, \\
& \bar{m}^{+}=\min _{1 \leq i \leq n} \min _{1 \leq j \leq m} \bar{\Delta}_{i j}^{+}, \\
& \stackrel{+}{M}=\max _{1 \leq i \leq n} \max _{1 \leq j \leq m} \underline{\Delta}_{i j}^{+}, \\
& \tilde{M}^{+}=\max _{1 \leq i \leq n} \max _{1 \leq j \leq m} \tilde{\Delta}_{i j}^{+}, \\
& \bar{M}^{+}=\max _{1 \leq i \leq n} \max _{1 \leq j \leq m} \bar{\Delta}_{i j}^{+}, \\
& G\left(x^{+}(\otimes), x_{i}(\otimes)\right)=\sum_{j=1}^{m} w_{i j} \gamma_{i j}^{+}, \quad i=1,2, \ldots, n,
\end{aligned}
$$

is called the three-parameter grey interval relational degree of the effect evaluation vector $x_{i}(\otimes)$ about the ideal optimal scheme effect evaluation vector $x^{+}(\otimes)$.
Definition 4. For three-parameter interval grey number $x_{i j}(\otimes) \in\left[\underline{x}_{i j}, \tilde{x}_{i j}, \bar{x}_{i j}\right]$,

$$
\gamma_{i j}^{-}=\frac{3}{5} \times \frac{\widetilde{m}^{-}+\varepsilon \tilde{M}^{-}}{\widetilde{\Delta}_{i j}^{-}+\varepsilon \tilde{M}^{-}}+\frac{2}{5} \times\left[(1-\delta) \frac{\underline{m}^{-}+\varepsilon \underline{M^{-}}}{\underline{\Delta}_{i j}^{-}+\varepsilon \underline{M}}+\delta \frac{\bar{m}^{-}}{\bar{\Delta}_{i j}^{-}+\varepsilon \bar{M}^{-}}\right] \text {, }
$$

is called the three-parameter grey interval relational coefficient of sub factor $x_{i j}$ with respect to ideal facto $x_{j}^{-}$. $\eta \in(0,1)$ is the resolution coefficient. $\delta \in(0,1)$ is the decision preference coefficient (according to [28] and experts' opinion, normally, $\varepsilon=\beta=0.5$ ), where

$$
\begin{aligned}
& \underline{\Delta}_{i j}^{-}=\left|\underline{x}_{i j}-\underline{x}_{j}^{-}\right|, \\
& \tilde{\Delta}_{i j}^{-}=\left|\tilde{x}_{i j}-\tilde{x}_{j}^{-}\right|, \quad i=1,2, \ldots, n ; j=1,2, \ldots, m, \\
& \bar{\Delta}_{i j}^{-}=\left|\bar{x}_{i j}-\bar{x}_{j}^{-}\right|, \\
& \underline{m}^{-}=\min _{1 \leq i \leq n} \min _{1 \leq j \leq m} \underline{\Delta}_{i j}^{-}, \\
& \tilde{m}^{-}=\min _{1 \leq i \leq n} \min _{1 \leq j \leq m} \tilde{\Delta}_{i j}^{-}, \\
& \bar{m}^{-}=\min _{1 \leq i \leq n} \min _{1 \leq j \leq m} \bar{\Delta}_{i j}^{-}, \\
& \underline{M}^{-}=\max _{1 \leq i \leq n} \max _{1 \leq j \leq m} \underline{\Delta}_{i j}^{-}, \\
& \tilde{M}^{-}=\max _{1 \leq i \leq n} \max _{1 \leq j \leq m} \tilde{\Delta}_{i j}^{-}, \\
& \bar{M}^{-}=\max _{1 \leq i \leq n} \max _{1 \leq j \leq m} \bar{\Delta}_{i j}^{-}, \\
& G\left(x^{-}(\otimes), x_{i}(\otimes)\right)=\sum_{j=1}^{m} w_{i j} \gamma_{i j}^{-}, \quad i=1,2, \ldots, n,
\end{aligned}
$$

is called the three-parameter grey interval relational degree of the effect evaluation vector $x_{i}(\otimes)$ about the critical scheme effect evaluation vector $x^{-}(\otimes)$.

5.3. Determination of Weight. In order to better reflect the balance and scientificity of expert evaluation and objective evaluation in the evaluation process, this paper adopts a combined weight method. Firstly, the triangular fuzzy analytical hierarchy process method is used to determine the subjective weight. Then the Gini coefficient method is used to determine the objective weight. Finally, the linear weighting method is used to combine the two methods, so as to increase its scientificity.

5.3.1. Determination of Weight Based on Triangular Fuzzy Analytic Hierarchy Process (TFAHP). Analytic hierarchy process (AHP) is a multicriteria decision analysis method 
which transforms subjective judgment into objective one. It makes people's subjective judgment process hierarchical and mathematical and provides a simple decision-making method for multiattribute decision-making problems.

Triangular fuzzy number has obvious advantages in solving the problems of complexity, fuzziness, and uncertainty. Fuzzy numbers have been studied extensively. [30-36] TFAHP (triangular fuzzy analytic hierarchy process) is a combination of fuzzy theory and AHP. It fully considers the subjective judgment of the evaluator, the fuzziness of decision, and the preference, which makes the decision result more objective and reasonable. The calculation steps are as follows:

(1) According to the above evaluation index system construction, the hierarchical model is established, as shown in Table 1. The top layer is target layer $A$. The middle layer is criterion layer $B$. The lowest layer is index layer $C$.

(2) Each expert can get the triangular fuzzy number judgment matrix of the target layer to the criterion layer and the criterion layer to the index layer by comparing each element. Let $\widetilde{x}_{i j}^{t}$ be the triangular fuzzy number judgment matrix of the expert $t$.Then, the triangular fuzzy number is $\widetilde{x}_{i j}^{t}=\left(\vec{l}_{i j}^{t}, \widetilde{m}_{i j}^{t}, \widetilde{u}_{i j}^{t}\right)$, where $\widetilde{l}_{i j}^{t}$ and $\widetilde{u}_{i j}^{t}$ are the upper and lower bounds of fuzzy numbers and $\widetilde{m}_{i j}^{t}$ is the fuzzy median. The smaller the value of $\widetilde{u}_{i j}^{t}-\widetilde{l}_{i j}^{t}$, the more fuzzy the judgment. When $\widetilde{u}_{i j}^{t}-\widetilde{l}_{i j}$ is 0 , the judgment is not fuzzy.

Each element value of the fuzzy judgment matrix represents the importance of one factor relative to another. Because it is difficult to construct the consistency matrix by $1-9$ and its reciprocal scaling method, the ranking result is far from the actual thinking 0.1-0.9 scale method which can better reflect the actual estimation of experts. Therefore, the $0.1-0.9$ scale method is used in this study, as shown in Table 2.

(3) The judgment matrix of each expert is synthesized, and the triangular fuzzy number in the judgment matrix is changed into nonfuzzy number. Suppose the total number of experts is $T$ and the weight of each expert is $q_{t}$; then, the synthetic judgment matrix is $\widetilde{X}=\sum_{t=1}^{T} q_{t} \widetilde{X}^{t}$.

According to the method of reference 35, the most likely estimated value of each factor in fuzzy judgment matrix $A$ by pairwise comparison are extracted. The fuzzy complementary judgment matrix $M: M=$ $\sum_{t=1}^{T} m_{i j}^{t} q_{t}$ is obtained. It can be transformed into fuzzy consistency matrix $M^{\prime}$ through formula $m_{i}^{\prime}=$ $\sum_{k=1}^{n} m_{i k}$ and $m_{i}^{\prime}=\left(\left(m_{i}^{\prime}-m_{j}^{\prime}\right) / 2(n-1)\right)+0.5$. If $M^{\prime}$ satisfies the requirement of consistency, then $\widetilde{X}$ also satisfies the requirement of consistency. For the fuzzy consistent matrix $M^{\prime}$ of $0.1-0.9$ scale, the consistency is checked by calculating $\delta$ and $\sigma$ to judge whether the fuzzy complementary judgment matrix is consistent with the actual situation:

$$
\begin{aligned}
& \delta=\max \left\{\left|m_{i j}^{\prime}-m_{i j}\right|\right\}, \\
& \sigma=\frac{\sqrt{\sum_{i=1}^{n} \sum_{j=1}^{n}\left(m_{i j}^{\prime}-m_{i j}\right)^{2}}}{n} .
\end{aligned}
$$

$\delta<0.2$ and $\sigma<0.1$, and the fuzzy matrix passes the consistency test, which is in line with the actual situation. Otherwise, the judgment matrix should be adjusted.

The fuzzy judgment matrix $\widetilde{X}$ is transformed into nonfuzzy matrix $F^{\prime \prime}$ :

$$
f^{\prime \prime}=\frac{l_{i j}}{2\left(l_{i j}+2\right)}+\frac{2 m_{i j}}{3}+\frac{u_{i j}}{6} .
$$

(4) In order to determine the index weight of each factor, the comprehensive importance of each element is calculated according to each fuzzy judgment matrix. It can be solved according to the weight formula:

$$
\omega_{i}=\frac{\sum_{j=1}^{n} f_{i j}^{\prime \prime}-0.5}{\sum\left(\sum_{j=1}^{n} f_{i j}^{\prime \prime}-0.5\right)} .
$$

\subsubsection{Weight Determination Method Based on Gini Coefficient}

(1) Principle of Gini Coefficient Weighting Method. The Gini coefficient weighting method is an objective weighting method by calculating Gini coefficient of evaluation index and normalizing Gini coefficient of each index. First of all, the different data of $n$ evaluation objects of a specific evaluation index can be regarded as the income of different level people. Then, the Gini coefficient of a certain index can be calculated. The value of Gini coefficient can reflect the data difference between different evaluation objects. Then, in order to ensure that weight of all indexes are in the range of 0 to 1 and the sum is 1, the Gini coefficient value of each index will be normalized to get the Gini coefficient weight of the evaluation index [37].

(2) Gini Coefficient Weight Calculation of Evaluation Index. We assume that $G_{k}$ is the Gini coefficient of the $k$ th index, $Y_{k i}$ is the $i$ th data of the $k$ th index, and $\mu K$ is the expected value of all data of the $k$ th index. Then, the Gini coefficient $G_{k}$ of the $k$ th index is shown as follows:

$$
\begin{aligned}
G_{k} & =\sum_{i=1}^{n} \sum_{j=1}^{n} \frac{\left|Y_{k i}-Y_{k j}\right|}{2 n^{2} \mu_{k}}, \\
G_{k} & =\sum_{i=1}^{n} \sum_{j=1}^{n} \frac{\left|Y_{k i}-Y_{k j}\right|}{\left(n^{2}-n\right)} .
\end{aligned}
$$

Especially, when the mean value of index data is not 0 , the Gini coefficient is calculated by the improved formula (14). When the mean value of the index data is 0 , the Gini 
TABLE 1: Index system hierarchical model of important nodes in the complex product design.

\begin{tabular}{lcccc}
\hline$A$ & \multicolumn{4}{c}{ Node importance evaluation } \\
\hline$B$ & Link degree $\left(B_{1}\right)$ & Average path length $\left(B_{2}\right)$ & Shortest route number $\left(B_{3}\right)$ & Value attributes of complex product $\left(B_{4}\right)$ \\
\hline$C$ & Clustering coefficient $\left(C_{1}\right)$ & Erdos $\left(C_{2}\right)$ & Node betweenness $\left(C_{3}\right)$ & Value of parts $\left(C_{4}\right)$ \\
\hline
\end{tabular}

TABLE 2: 0.1-0.9 quantitative scale.

\begin{tabular}{lc}
\hline Scale & Meaning \\
\hline 0.1 & When one factor is compared with another, the latter is extremely important \\
0.2 & When one factor is compared with another, the latter is strongly important \\
0.3 & When one factor is compared with another, the latter is obviously important \\
0.4 & When one factor is compared with another, the latter is slightly important \\
0.5 & When one factor is compared with another, the two factors are of equal importance \\
0.6 & When one factor is compared with another, the former is slightly more important \\
0.7 & When one factor is compared with another, the former is obviously important \\
0.8 & When one factor is compared with another, the former is strongly important \\
0.9 & When one factor is compared with another, the former is extremely important \\
\hline
\end{tabular}

coefficient of the index is calculated by the original formula (15). The Gini coefficient of the index truly reflects the data changes of different evaluation objects of the index.

Gini coefficient weight $g_{k}$ of the $k$ th index can be obtained by normalizing the Gini coefficient value of each index:

$$
g_{k}=\frac{G_{k}}{\sum_{i=1}^{m} G_{i}}
$$

where $g_{k}$ is Gini coefficient weight of the $k$ th index, $G_{k}$ is Gini coefficient value of the $k$ th index, and $m$ is the number of indexes.

The advantages of the Gini coefficient weighting method are as follows. First, the weight calculation is not affected by the unit dimension of the index, and the definition of Gini coefficient itself eliminates the dimensional influence. Second, the Gini coefficient value of the evaluation index reflects the difference between any two evaluation objects. Gini coefficient weight reflects the difference between the data of different evaluation objects of an index. And, the weight reflects the data information of the index, which meets the requirements of the objective weighting method.

\subsubsection{Combination Weighting Method Based on TFAHP-} Gini Coefficient. In order to be more scientific, we adopt the linear weighting method to combine the two methods after the weight determining based on subjective and objective.

The weight vector of linear combination is calculated and proved as follows.

Suppose $N$ kinds of subjective and objective methods to get $l$ kinds of weights. The weight vectors are $W_{1}, W_{2}, \ldots$, $W_{L}$. The $k$ th weight direction quantity is $W^{k}=\left(\omega_{1}^{k}, \omega_{2}^{k}, \ldots\right.$, $\left.\Phi_{m}^{k}\right)$. It satisfies that $\sum_{j=1}^{m} \Phi_{j}^{k}=1, \Phi_{j}^{k} \geq 0(j=1,2, \ldots$, $m ; k=1,2, \ldots, L)$. The vector obtained by linear combination of multiple weight vectors is called linear combination weight vector. Let $W^{*}=\left(\omega_{1}^{*}, \omega_{2}^{*}, \ldots, \varpi_{m}^{*}\right)^{T}$ be the linear combination weight vector of $L$ weight vectors. It can be expressed in the vector form as $W^{*}=\sum_{k=1}^{L} x_{k} w^{k}$, where $x_{k}$ is the linear combination coefficient. It satisfies $\sum_{k=1}^{L} x_{k}=1, x_{k} \geq 0$. The solution of linear combined weight vector is a multiobjective optimization problem. On the one hand, the sum of weighted generalized distances between all schemes and ideal schemes should be minimized. On the other hand, the uncertainty of combination coefficient should be eliminated as far as possible. According to the Jaynes maximum entropy principle, the comprehensive weight coefficient of the index should be determined so that the Shannon entropy takes the maximum value. Therefore, the following optimization decision model is established:

$$
\begin{aligned}
& \min \left[\mu \sum_{i=1}^{n} \sum_{j=1}^{m} \sum_{k=1}^{L} x_{k} \Phi_{j}^{k}\left(1-r_{i j}\right)+(1-\mu) \sum x_{k} \ln x_{k}\right], \\
& \text { s.t. } \quad \sum_{k=1}^{L} x_{k}=1, \quad x_{k} \geq 0,
\end{aligned}
$$

where $r_{i j}$ is the relational coefficient, $0 \leq \mu \leq 1$. It can be used to express the balance coefficient between two targets, which can be given in advance according to practical problems.

According to [3], there is a unique solution for the single objective optimization problem (SP). The solution is as follows:

$$
x=\left[\frac{s_{1}}{\sum_{k=1}^{l} s_{k}}, \frac{s_{2}}{\sum_{k=1}^{l} s_{k}}, \ldots, \frac{s_{L}}{\sum_{k=1}^{L} s_{k}}\right]
$$

where $\quad s_{k}=\exp \left\{-\left[1+\mu \sum_{i=1}^{n} \sum_{j=1}^{m} \quad\left(\omega_{j}^{k}\left(1-r_{i j}\right) /(1-\mu)\right)\right\}\right.$ $(k=1,2, \ldots, L), N$ is the number of evaluation schemes, and $M$ is the number of evaluation indexes. 


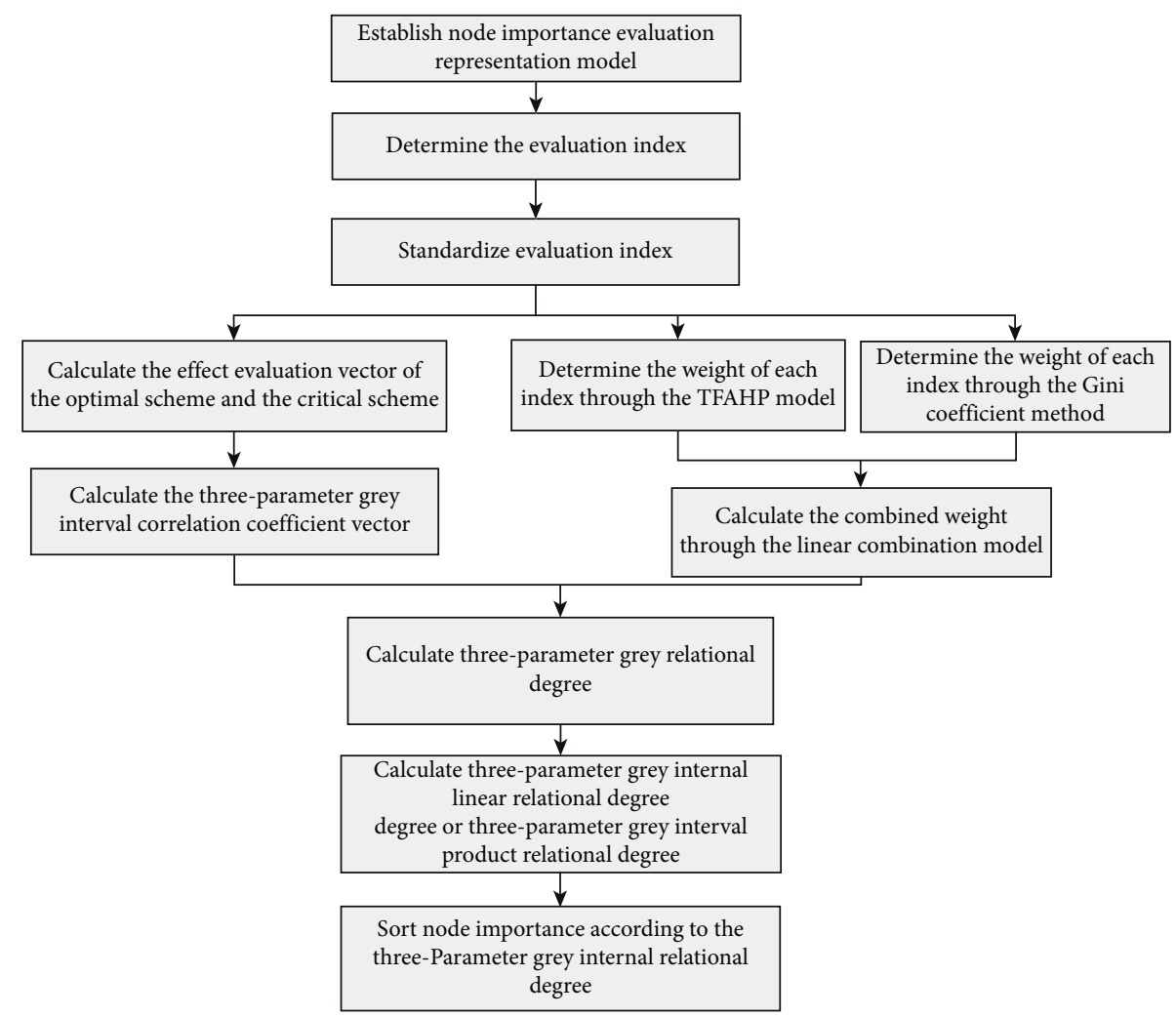

FIgURE 3: Three-parameter interval grey number grey relational evaluation model algorithm.

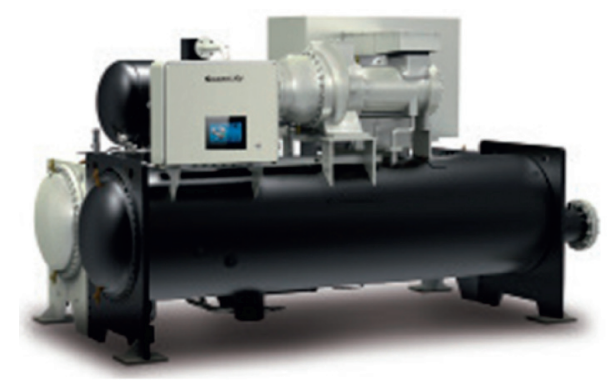

FIGURE 4: Large permanent magnet synchronous centrifugal unit.

5.3.4. Steps of Complex Product Node Importance Evaluation Based on Three-Parameter Grey Relational Degree. To sum up, the three-parameter interval grey relational evaluation algorithm of complex product node importance is as follows:

Step 1: construct the model of complex product presentation based on the multilayer complex network model.

Step 2: determine the evaluation index.

Step 3: standardize the original three-parameter interval grey number effect evaluation index, and obtain the standardized effect evaluation vector formula of each scheme.

Step 4: solve the ideal optimal solution for the decisionmaking problem and the effect evaluation vector $x^{+}(\otimes)$ and the critical solution $x^{-}(\otimes)$.

Step 5: obtain the three-parameter grey interval relational coefficient vector of each scheme, the ideal scheme and the critical scheme.
TABle 3: Main parts and node name of permanent magnet synchronous centrifugal compressor.

\begin{tabular}{lc}
\hline Node & Parts \\
\hline$V_{1}$ & Principal axis \\
$V_{2}$ & Inspiratory chamber \\
$V_{3}$ & Import regulation structure \\
$V_{4}$ & Impeller \\
$V_{5}$ & Diffuser \\
$V_{6}$ & Volute \\
$V_{7}$ & Retaining ring \\
$V_{8}$ & Tight ring \\
$V_{9}$ & Thrust disk \\
$V_{10}$ & Separator \\
$V_{11}$ & Balance plate \\
$V_{12}$ & Refluxer \\
$V_{13}$ & Sealing \\
$V_{14}$ & Bend \\
$V_{15}$ & Bearing \\
$V_{16}$ & Electrical machine \\
$V_{17}$ & Lubricated gears \\
$V_{18}$ & Bearing with lubrication \\
$V_{19}$ & Curved casing \\
$V_{20}$ & Pressure-regulating valve \\
$V_{21}$ & Temperature sensor \\
$V_{22}$ & Extraction cooler \\
$V_{23}$ & Pressure sensor \\
$V_{24}$ & Evaporator \\
$V_{25}$ & Return evaporator \\
$V_{26}$ & Lubricating oil \\
$V_{27}$ & Cryogen \\
$V_{28}$ & Oil cooler \\
$V_{29}$ & Electronic expansion valve \\
$V_{30}$ & Drier filter \\
$V_{31}$ & Condenser \\
\hline
\end{tabular}


TABLE 4: Function knowledge network connection information.

\begin{tabular}{|c|c|c|c|c|c|c|c|c|}
\hline \multicolumn{9}{|c|}{ Function knowledge network } \\
\hline Source & Target & Weight & Source & Target & Weight & Source & Target & Weight \\
\hline 1 & 4 & 0.52 & 9 & 21 & 0.27 & 18 & 23 & 0.39 \\
\hline 1 & 7 & 0.59 & 9 & 22 & 0.50 & 18 & 24 & 0.65 \\
\hline 1 & 8 & 0.56 & 10 & 11 & 0.93 & 18 & 25 & 0.70 \\
\hline 1 & 9 & 0.28 & 10 & 12 & 0.59 & 18 & 29 & 0.52 \\
\hline 1 & 11 & 0.56 & 10 & 13 & 0.52 & 19 & 20 & 0.12 \\
\hline 1 & 15 & 0.46 & 10 & 14 & 0.48 & 19 & 21 & 0.48 \\
\hline 1 & 17 & 0.56 & 10 & 15 & 0.58 & 19 & 22 & 0.56 \\
\hline 1 & 18 & 0.69 & 11 & 13 & 0.55 & 19 & 23 & 0.23 \\
\hline 2 & 3 & 0.45 & 11 & 15 & 0.50 & 19 & 25 & 0.67 \\
\hline 2 & 5 & 0.44 & 11 & 16 & 0.62 & 19 & 28 & 0.56 \\
\hline 2 & 6 & 0.49 & 11 & 17 & 0.40 & 20 & 23 & 0.47 \\
\hline 2 & 12 & 0.44 & 11 & 20 & 0.58 & 20 & 29 & 0.39 \\
\hline 3 & 4 & 0.25 & 11 & 21 & 0.55 & 20 & 31 & 0.58 \\
\hline 3 & 5 & 0.25 & 12 & 13 & 0.64 & 21 & 22 & 0.43 \\
\hline 3 & 9 & 0.42 & 12 & 14 & 0.56 & 21 & 23 & 0.58 \\
\hline 3 & 12 & 0.69 & 12 & 15 & 0.35 & 21 & 24 & 0.60 \\
\hline 3 & 14 & 0.54 & 12 & 16 & 0.60 & 21 & 27 & 0.22 \\
\hline 4 & 5 & 0.66 & 13 & 14 & 0.51 & 21 & 28 & 0.68 \\
\hline 4 & 6 & 0.59 & 13 & 15 & 0.52 & 21 & 29 & 0.36 \\
\hline 4 & 11 & 0.27 & 13 & 16 & 0.61 & 21 & 30 & 0.54 \\
\hline 4 & 12 & 0.44 & 13 & 21 & 0.41 & 21 & 31 & 0.41 \\
\hline 4 & 14 & 0.36 & 13 & 23 & 0.44 & 22 & 24 & 0.45 \\
\hline 4 & 21 & 0.19 & 14 & 15 & 0.49 & 22 & 25 & 0.20 \\
\hline 4 & 23 & 0.57 & 14 & 16 & 0.32 & 22 & 27 & 0.35 \\
\hline 5 & 6 & 0.31 & 14 & 19 & 0.29 & 22 & 28 & 0.52 \\
\hline 5 & 7 & 0.48 & 14 & 25 & 0.68 & 22 & 29 & 0.43 \\
\hline 5 & 8 & 0.50 & 15 & 16 & 0.55 & 22 & 30 & 0.38 \\
\hline 5 & 10 & 0.39 & 15 & 20 & 0.37 & 22 & 31 & 0.38 \\
\hline 5 & 23 & 0.58 & 15 & 23 & 0.31 & 23 & 27 & 0.42 \\
\hline 6 & 7 & 0.67 & 16 & 19 & 0.46 & 23 & 28 & 0.23 \\
\hline 6 & 8 & 0.69 & 16 & 20 & 0.37 & 24 & 25 & 0.21 \\
\hline 6 & 10 & 0.60 & 16 & 21 & 0.25 & 24 & 27 & 0.30 \\
\hline 6 & 12 & 0.63 & 16 & 23 & 0.37 & 24 & 28 & 0.60 \\
\hline 6 & 14 & 0.58 & 16 & 29 & 0.55 & 24 & 29 & 0.40 \\
\hline 7 & 8 & 0.59 & 17 & 18 & 0.59 & 24 & 30 & 0.51 \\
\hline 7 & 9 & 0.54 & 17 & 19 & 0.29 & 24 & 31 & 0.57 \\
\hline 7 & 10 & 0.68 & 17 & 20 & 0.61 & 25 & 27 & 0.35 \\
\hline 7 & 11 & 0.63 & 17 & 21 & 0.20 & 25 & 28 & 0.49 \\
\hline 7 & 13 & 0.72 & 17 & 23 & 0.69 & 25 & 30 & 0.51 \\
\hline 7 & 14 & 0.51 & 17 & 24 & 0.68 & 25 & 31 & 0.40 \\
\hline 8 & 11 & 0.22 & 17 & 25 & 0.40 & 26 & 29 & 0.25 \\
\hline 8 & 12 & 0.52 & 17 & 28 & 0.19 & 27 & 28 & 0.17 \\
\hline 8 & 13 & 0.81 & 17 & 31 & 0.56 & 27 & 30 & 0.40 \\
\hline 9 & 11 & 0.27 & 18 & 19 & 0.47 & 27 & 31 & 0.63 \\
\hline 9 & 12 & 0.41 & 18 & 20 & 0.39 & 28 & 31 & 0.42 \\
\hline \multirow[t]{2}{*}{9} & 14 & 0.51 & 18 & 21 & 0.61 & 29 & 30 & 0.35 \\
\hline & & & & & & 30 & 31 & 0.68 \\
\hline
\end{tabular}

Step 6: solve the TFAHP and Gini coefficient models, combine the weights, and obtain the weight of each scheme under different attributes.

Step 7: calculate the three-parameter interval grey number relational degree $G\left(x^{+}(\otimes), x_{i}(\otimes)\right)$ and $G\left(x^{-}(\otimes), x_{i}(\otimes)\right)$ $(i=1,2, \ldots, n)$ between each scheme and ideal scheme and critical scheme, and calculate the three-parameter grey interval linear relational degree or three-parameter grey interval product relational degree $G\left(x_{i}(\otimes)\right)(i=$ $1,2, \ldots, n)$ of each scheme.
Step 8: the schemes are sorted according to the relevance degree $G\left(x_{i}(\otimes)\right)(i=1,2, \ldots, n)$. The scheme corresponding to the maximum relational degree is the optimal one.

The flow of this algorithm is shown in Figure 3.

\section{Case Study}

The high-speed permanent magnet synchronous frequency conversion centrifugal high-power chiller of $G$ 
TABLE 5: Behavior knowledge network connection information.

\begin{tabular}{|c|c|c|c|c|c|c|c|c|}
\hline \multicolumn{9}{|c|}{ Behavior knowledge network } \\
\hline Source & Target & Weight & Source & Target & Weight & Source & Target & Weight \\
\hline 1 & 6 & 0.45 & 9 & 11 & 0.34 & 17 & 21 & 0.63 \\
\hline 1 & 7 & 0.34 & 9 & 12 & 0.27 & 17 & 23 & 0.50 \\
\hline 1 & 8 & 0.43 & 9 & 13 & 0.37 & 17 & 24 & 0.53 \\
\hline 1 & 9 & 0.56 & 9 & 21 & 0.44 & 17 & 25 & 0.79 \\
\hline 1 & 11 & 0.28 & 9 & 23 & 0.60 & 18 & 19 & 0.29 \\
\hline 1 & 15 & 0.33 & 10 & 11 & 0.64 & 18 & 20 & 0.60 \\
\hline 2 & 3 & 0.53 & 10 & 13 & 0.56 & 18 & 21 & 0.26 \\
\hline 2 & 4 & 0.50 & 10 & 14 & 0.22 & 18 & 25 & 0.45 \\
\hline 2 & 6 & 0.16 & 10 & 15 & 0.32 & 19 & 20 & 0.79 \\
\hline 2 & 13 & 0.43 & 11 & 13 & 0.49 & 19 & 21 & 0.46 \\
\hline 3 & 4 & 0.37 & 11 & 15 & 0.46 & 19 & 22 & 0.23 \\
\hline 3 & 5 & 0.53 & 11 & 16 & 0.32 & 19 & 25 & 0.66 \\
\hline 3 & 9 & 0.62 & 11 & 17 & 0.31 & 20 & 23 & 0.49 \\
\hline 3 & 12 & 0.50 & 11 & 20 & 0.36 & 20 & 29 & 0.28 \\
\hline 3 & 14 & 0.52 & 11 & 21 & 0.66 & 21 & 22 & 0.32 \\
\hline 4 & 5 & 0.22 & 12 & 13 & 0.50 & 21 & 23 & 0.59 \\
\hline 4 & 6 & 0.52 & 12 & 14 & 0.42 & 21 & 24 & 0.50 \\
\hline 4 & 11 & 0.47 & 12 & 15 & 0.51 & 21 & 30 & 0.45 \\
\hline 4 & 12 & 0.67 & 12 & 16 & 0.29 & 21 & 31 & 0.26 \\
\hline 4 & 13 & 0.59 & 13 & 14 & 0.26 & 22 & 24 & 0.46 \\
\hline 4 & 20 & 0.61 & 13 & 15 & 0.69 & 22 & 25 & 0.27 \\
\hline 4 & 21 & 0.64 & 13 & 16 & 0.53 & 22 & 29 & 0.23 \\
\hline 5 & 6 & 0.63 & 13 & 21 & 0.36 & 22 & 30 & 0.68 \\
\hline 5 & 7 & 0.59 & 13 & 23 & 0.53 & 22 & 31 & 0.24 \\
\hline 5 & 8 & 0.56 & 14 & 15 & 0.21 & 23 & 27 & 0.33 \\
\hline 5 & 9 & 0.26 & 14 & 16 & 0.30 & 23 & 28 & 0.41 \\
\hline 5 & 21 & 0.21 & 14 & 19 & 0.53 & 24 & 25 & 0.34 \\
\hline 6 & 7 & 0.61 & 14 & 25 & 0.58 & 24 & 27 & 0.42 \\
\hline 6 & 9 & 0.51 & 15 & 16 & 0.63 & 24 & 29 & 0.76 \\
\hline 6 & 10 & 0.67 & 15 & 20 & 0.61 & 24 & 30 & 0.41 \\
\hline 6 & 12 & 0.69 & 15 & 23 & 0.70 & 24 & 31 & 0.31 \\
\hline 6 & 13 & 0.46 & 16 & 19 & 0.67 & 25 & 27 & 0.51 \\
\hline 7 & 8 & 0.33 & 16 & 20 & 0.26 & 25 & 28 & 0.32 \\
\hline 7 & 9 & 0.44 & 16 & 21 & 0.23 & 25 & 31 & 0.36 \\
\hline 7 & 11 & 0.23 & 16 & 22 & 0.61 & 26 & 29 & 0.56 \\
\hline 7 & 12 & 0.40 & 16 & 28 & 0.21 & 27 & 30 & 0.42 \\
\hline 7 & 13 & 0.39 & 17 & 18 & 0.66 & 27 & 31 & 0.50 \\
\hline 7 & 14 & 0.61 & 16 & 28 & 0.21 & 28 & 31 & 0.57 \\
\hline 8 & 10 & 0.34 & 17 & 18 & 0.66 & 29 & 30 & 0.46 \\
\hline 8 & 12 & 0.35 & 17 & 19 & 0.49 & 30 & 31 & 0.37 \\
\hline 8 & 13 & 0.41 & 17 & 20 & 0.31 & & & \\
\hline
\end{tabular}

enterprise has been unanimously recognized as the world's first high-speed and high-power word synchronous frequency conversion centrifugal chiller after being certified by many experts. Its technology has reached the international leading level. Its design process will involve many parts, among which the parts have complex connections in many aspects. In this paper, multilayer network is used to sort out its relationship in detail, and the identification of key design parts is studied. The product drawing and component composition are shown in Figure 4 and Table 3.
First of all, combined with enterprise product knowledge base, design base, case base, and interviews with designers, we analyze the relationship between functional behavior and structure network of the large permanent magnet synchronous centrifugal unit. The multilayer network knowledge association is shown from Tables 4 to 6 .

Through the calculation of index system, we can get the three-parameter interval grey number of the evaluation index as follows. This paper combines the enterprise product knowledge base, design library, and case library, as well as interviews with designers, to analyze the relationship and the 
TABLE 6: Structure knowledge network connection information.

\begin{tabular}{|c|c|c|c|c|c|c|c|c|}
\hline \multicolumn{9}{|c|}{ Structure knowledge network } \\
\hline Source & Target & Weight & Source & Target & Weight & Source & Target & Weight \\
\hline 1 & 4 & 0.23 & 10 & 12 & 0.49 & 18 & 29 & 0.21 \\
\hline 1 & 7 & 0.31 & 10 & 13 & 0.50 & 19 & 20 & 0.30 \\
\hline 1 & 8 & 0.29 & 10 & 15 & 0.33 & 19 & 21 & 0.79 \\
\hline 1 & 9 & 0.50 & 11 & 13 & 0.32 & 19 & 22 & 0.33 \\
\hline 1 & 11 & 0.46 & 11 & 15 & 0.52 & 19 & 25 & 0.66 \\
\hline 1 & 18 & 0.52 & 11 & 16 & 0.30 & 19 & 28 & 0.42 \\
\hline 2 & 3 & 0.44 & 11 & 17 & 0.47 & 20 & 23 & 0.38 \\
\hline 2 & 5 & 0.57 & 11 & 20 & 0.65 & 20 & 31 & 0.20 \\
\hline 3 & 4 & 0.46 & 12 & 13 & 0.40 & 21 & 23 & 0.44 \\
\hline 3 & 5 & 0.63 & 12 & 14 & 0.54 & 21 & 24 & 0.59 \\
\hline 3 & 9 & 0.44 & 12 & 15 & 0.68 & 21 & 27 & 0.14 \\
\hline 3 & 14 & 0.21 & 13 & 14 & 0.63 & 21 & 28 & 0.11 \\
\hline 4 & 5 & 0.59 & 13 & 15 & 0.66 & 21 & 30 & 0.23 \\
\hline 4 & 6 & 0.45 & 13 & 16 & 0.28 & 21 & 31 & 0.53 \\
\hline 4 & 11 & 0.41 & 13 & 23 & 0.29 & 22 & 24 & 0.44 \\
\hline 4 & 12 & 0.40 & 14 & 15 & 0.79 & 22 & 25 & 0.46 \\
\hline 4 & 23 & 0.48 & 14 & 16 & 0.48 & 22 & 27 & 0.41 \\
\hline 5 & 6 & 0.31 & 14 & 19 & 0.58 & 22 & 28 & 0.65 \\
\hline 5 & 7 & 0.35 & 14 & 25 & 0.34 & 22 & 30 & 0.71 \\
\hline 5 & 9 & 0.30 & 15 & 16 & 0.31 & 22 & 31 & 0.49 \\
\hline 5 & 23 & 0.28 & 15 & 20 & 0.46 & 23 & 27 & 0.42 \\
\hline 6 & 7 & 0.38 & 15 & 23 & 0.24 & 23 & 28 & 0.45 \\
\hline 6 & 8 & 0.65 & 16 & 19 & 0.70 & 24 & 25 & 0.75 \\
\hline 6 & 12 & 0.28 & 16 & 20 & 0.67 & 24 & 28 & 0.87 \\
\hline 6 & 14 & 0.22 & 16 & 21 & 0.23 & 24 & 29 & 0.49 \\
\hline 7 & 8 & 0.47 & 16 & 23 & 0.54 & 24 & 30 & 0.35 \\
\hline 7 & 9 & 0.61 & 16 & 29 & 0.43 & 24 & 31 & 0.49 \\
\hline 7 & 10 & 0.61 & 17 & 18 & 0.41 & 25 & 27 & 0.47 \\
\hline 7 & 11 & 0.38 & 17 & 19 & 0.44 & 25 & 30 & 0.33 \\
\hline 7 & 14 & 0.43 & 17 & 20 & 0.67 & 25 & 31 & 0.55 \\
\hline 8 & 11 & 0.47 & 17 & 21 & 0.54 & 26 & 29 & 0.56 \\
\hline 8 & 12 & 0.38 & 17 & 25 & 0.28 & 27 & 28 & 0.19 \\
\hline 8 & 13 & 0.46 & 17 & 28 & 0.62 & 27 & 30 & 0.45 \\
\hline 9 & 11 & 0.36 & 17 & 31 & 0.26 & 27 & 31 & 0.87 \\
\hline 9 & 12 & 0.46 & 18 & 19 & 0.60 & 28 & 31 & 0.68 \\
\hline 9 & 14 & 0.44 & 18 & 20 & 0.55 & 29 & 30 & 0.56 \\
\hline 9 & 22 & 0.52 & 18 & 23 & 0.31 & 30 & 31 & 0.39 \\
\hline 10 & 11 & 0.55 & 18 & 24 & 0.45 & & & \\
\hline
\end{tabular}

FBS complex network between the parts of the large permanent magnet synchronous centrifugal unit. Then, the network characteristic analysis is carried out. According to the internal expert experience of the enterprise, the FBS multilayer network is assigned $(1 / 3,1 / 3,1 / 3),(1 / 2,1 / 4,1 / 4)$, and $(1 / 2,1 / 4), 1 / 4)$ for obtaining three-parameter index data. The three-parameter value of the value information of the node is obtained through enterprise research. Then, we can get the three-parameter interval grey number of the evaluation index as follows: 


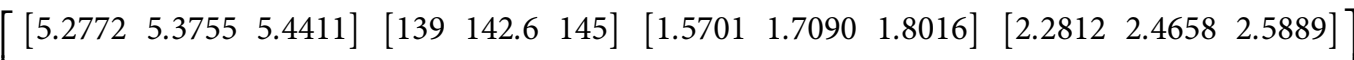

$$
\begin{aligned}
& {\left[\begin{array}{lll}
3.6959 & 4.0257 & 4.2455
\end{array}\right]\left[\begin{array}{lll}
43 & 47.2 & 50
\end{array}\right]\left[\begin{array}{llll}
2.9399 & 3.2129 & 3.3948
\end{array}\right]\left[\begin{array}{llll}
0.7282 & 0.7512 & 0.7665
\end{array}\right]} \\
& {\left[\begin{array}{lll}
3.8358 & 4.4389 & 4.8409
\end{array}\right] \quad\left[\begin{array}{lll}
87 & 90 & 92
\end{array}\right] \quad\left[\begin{array}{llll}
3.5253 & 3.7895 & 3.9656
\end{array}\right]\left[\begin{array}{llll}
0.8195 & 0.8455 & 0.8628
\end{array}\right]} \\
& {\left[\begin{array}{lll}
4.2019 & 4.3726 & 4.4863
\end{array}\right]\left[\begin{array}{lll}
128 & 131.6 & 134
\end{array}\right]\left[\begin{array}{llll}
3.4982 & 3.7958 & 3.9942
\end{array}\right]\left[\begin{array}{llll}
2.0347 & 2.1363 & 2.2040
\end{array}\right]} \\
& {\left[\begin{array}{lll}
2.0656 & 2.5827 & 2.9274
\end{array}\right] \quad\left[\begin{array}{lll}
31 & 33.4 & 35
\end{array}\right] \quad\left[\begin{array}{llll}
3.3349 & 3.6814 & 3.9124
\end{array}\right]\left[\begin{array}{llll}
0.3746 & 0.3837 & 0.3898
\end{array}\right]} \\
& {\left[\begin{array}{lll}
3.5186 & 3.4648 & 3.4290
\end{array}\right]\left[\begin{array}{lll}
32 & 36.2 & 39
\end{array}\right]\left[\begin{array}{llll}
3.1801 & 3.3571 & 3.4751
\end{array}\right]\left[\begin{array}{llll}
0.3470 & 0.3683 & 0.3825
\end{array}\right]} \\
& {\left[\begin{array}{lll}
3.2530 & 3.6687 & 3.9458
\end{array}\right] \quad\left[\begin{array}{lll}
30 & 34.2 & 37
\end{array}\right]\left[\begin{array}{llll}
2.7420 & 3.0989 & 3.3368
\end{array}\right]\left[\begin{array}{lll}
0.9096 & 0.9405 & 0.9611
\end{array}\right]} \\
& {\left[\begin{array}{lll}
3.6148 & 3.7531 & 3.8453
\end{array}\right]\left[\begin{array}{lll}
21 & 25.2 & 28
\end{array}\right]\left[\begin{array}{lll}
2.0211 & 2.4675 & 2.7651
\end{array}\right]\left[\begin{array}{llll}
0.1458 & 0.4538 & 0.6591
\end{array}\right]} \\
& {\left[\begin{array}{lll}
3.5515 & 3.7544 & 3.8896
\end{array}\right]\left[\begin{array}{lll}
26 & 29.6 & 32
\end{array}\right] \quad\left[\begin{array}{llll}
3.6857 & 3.8382 & 3.9398
\end{array}\right]\left[\begin{array}{llll}
0.9985 & 0.8067 & 0.6788
\end{array}\right]} \\
& {\left[\begin{array}{lll}
1.2876 & 1.8667 & 2.2528
\end{array}\right]\left[\begin{array}{lll}
23 & 27.8 & 31
\end{array}\right]\left[\begin{array}{lll}
3.8336 & 4.1062 & 4.2879
\end{array}\right]\left[\begin{array}{llll}
0.5379 & 0.5567 & 0.5692
\end{array}\right]} \\
& {\left[\begin{array}{lll}
2.3693 & 2.7321 & 2.9740
\end{array}\right] \quad\left[\begin{array}{lll}
36 & 39 & 41
\end{array}\right] \quad\left[\begin{array}{llll}
3.3977 & 3.8290 & 4.1166
\end{array}\right]\left[\begin{array}{llll}
0.8360 & 0.8581 & 0.8728
\end{array}\right]} \\
& {\left[\begin{array}{lll}
2.8436 & 3.2467 & 3.5154
\end{array}\right] \quad\left[\begin{array}{lll}
38 & 41 & 43
\end{array}\right] \quad\left[\begin{array}{llll}
2.7009 & 3.1348 & 3.4240
\end{array}\right]\left[\begin{array}{llll}
0.7279 & 0.7702 & 0.7985
\end{array}\right]} \\
& {\left[\begin{array}{lll}
2.1737 & 2.6398 & 2.9506
\end{array}\right] \quad\left[\begin{array}{lll}
32 & 35 & 37
\end{array}\right] \quad\left[\begin{array}{llll}
3.4680 & 3.6469 & 3.7662
\end{array}\right]\left[\begin{array}{llll}
0.9241 & 0.9612 & 0.9859
\end{array}\right]} \\
& {\left[\begin{array}{lll}
2.4560 & 2.7803 & 2.9964
\end{array}\right] \quad\left[\begin{array}{lll}
26 & 29 & 31
\end{array}\right] \quad\left[\begin{array}{llll}
3.6845 & 3.8422 & 3.9473
\end{array}\right]\left[\begin{array}{llll}
0.7017 & 0.7702 & 0.8158
\end{array}\right]} \\
& {\left[\begin{array}{lll}
1.9852 & 2.1486 & 2.2574
\end{array}\right]\left[\begin{array}{lllll}
35 & 40.4 & 44
\end{array}\right]\left[\begin{array}{llll}
4.6799 & 4.8129 & 4.9015
\end{array}\right]\left[\begin{array}{llll}
0.8126 & 0.8492 & 0.8737
\end{array}\right]} \\
& X(\otimes)_{O}=\left[\begin{array}{lll}
2.6777 & 3.2085 & 3.5624
\end{array}\right] \quad\left[\begin{array}{lll}
29 & 32 & 34
\end{array}\right] \quad\left[\begin{array}{lllll}
3.1567 & 3.5532 & 3.8175
\end{array}\right]\left[\begin{array}{llll}
0.3707 & 0.3947 & 0.4107
\end{array}\right] \text {. } \\
& {\left[\begin{array}{lll}
1.4577 & 2.1785 & 2.6590
\end{array}\right] \quad\left[\begin{array}{lll}
26 & 27.8 & 29
\end{array}\right]\left[\begin{array}{llll}
3.0778 & 3.4801 & 3.7483
\end{array}\right]\left[\begin{array}{llll}
0.6101 & 0.6628 & 0.6980
\end{array}\right]} \\
& {\left[\begin{array}{lll}
3.9226 & 4.2512 & 4.4703
\end{array}\right] \quad\left[\begin{array}{lll}
76 & 83.8 & 89
\end{array}\right]\left[\begin{array}{llll}
2.9147 & 3.2387 & 3.4546
\end{array}\right]\left[\begin{array}{llll}
1.0031 & 1.1734 & 1.2869
\end{array}\right]} \\
& {\left[\begin{array}{lll}
4.6784 & 4.9707 & 5.1655
\end{array}\right] \quad\left[\begin{array}{lll}
78 & 86.4 & 92
\end{array}\right]\left[\begin{array}{llll}
3.6177 & 4.0368 & 4.3161
\end{array}\right]\left[\begin{array}{llll}
1.0211 & 1.1884 & 1.2999
\end{array}\right]} \\
& {\left[\begin{array}{lll}
2.0672 & 2.5348 & 2.8466
\end{array}\right]\left[\begin{array}{lll}
30 & 33.6 & 36
\end{array}\right]\left[\begin{array}{lll}
3.9365 & 4.2157 & 4.4019
\end{array}\right]\left[\begin{array}{llll}
0.5793 & 0.5898 & 0.5968
\end{array}\right]} \\
& {\left[\begin{array}{lll}
2.5180 & 2.7985 & 2.9856
\end{array}\right]\left[\begin{array}{lll}
35 & 38.6 & 41
\end{array}\right]\left[\begin{array}{llll}
4.0827 & 4.5355 & 4.8373
\end{array}\right]\left[\begin{array}{llll}
0.4204 & 0.4333 & 0.4420
\end{array}\right]} \\
& {\left[\begin{array}{lll}
2.9706 & 3.4396 & 3.7522
\end{array}\right] \quad\left[\begin{array}{lll}
37 & 40.6 & 43
\end{array}\right] \quad\left[\begin{array}{llll}
3.4184 & 3.6110 & 3.7395
\end{array}\right]\left[\begin{array}{llll}
0.4923 & 0.5040 & 0.5118
\end{array}\right]} \\
& {\left[\begin{array}{lll}
2.6569 & 2.8298 & 2.9451
\end{array}\right] \quad\left[\begin{array}{lll}
36 & 39.6 & 42
\end{array}\right]\left[\begin{array}{llll}
3.8562 & 4.0728 & 4.2173
\end{array}\right]\left[\begin{array}{llll}
0.4735 & 0.5034 & 0.5234
\end{array}\right]} \\
& {\left[\begin{array}{lll}
3.9019 & 4.4699 & 4.8486
\end{array}\right]\left[\begin{array}{lll}
55 & 58.6 & 61
\end{array}\right]\left[\begin{array}{llll}
2.9948 & 3.4043 & 3.6773
\end{array}\right]\left[\begin{array}{llll}
0.8101 & 0.8383 & 0.8572
\end{array}\right]} \\
& {\left[\begin{array}{lll}
3.1264 & 3.5869 & 3.8939
\end{array}\right]\left[\begin{array}{lll}
34 & 38.2 & 41
\end{array}\right]\left[\begin{array}{llll}
3.3830 & 3.7307 & 3.9625
\end{array}\right]\left[\begin{array}{llll}
0.7072 & 0.7874 & 0.8409
\end{array}\right]} \\
& {\left[\begin{array}{lll}
3.6097 & 4.1982 & 4.5906
\end{array}\right]\left[\begin{array}{lll}
30 & 34.2 & 37
\end{array}\right]\left[\begin{array}{llll}
3.3496 & 3.5309 & 3.6517
\end{array}\right]\left[\begin{array}{llll}
0.4995 & 0.5218 & 0.5367
\end{array}\right]} \\
& {\left[\begin{array}{lll}
3.8147 & 4.0691 & 4.2386
\end{array}\right] \quad\left[\begin{array}{lll}
29 & 32 & 34
\end{array}\right] \quad\left[\begin{array}{lll}
3.3914 & 4.1287 & 4.6202
\end{array}\right]\left[\begin{array}{llll}
0.5435 & 0.5653 & 0.5799
\end{array}\right]} \\
& {\left[\begin{array}{lll}
2.8457 & 3.2798 & 3.5692
\end{array}\right]\left[\begin{array}{lll}
28 & 30.4 & 32
\end{array}\right]\left[\begin{array}{llll}
3.3991 & 3.5278 & 3.6136
\end{array}\right]\left[\begin{array}{llll}
0.4307 & 0.4819 & 0.5161
\end{array}\right]} \\
& {\left[\begin{array}{lll}
3.1386 & 3.8243 & 4.2814
\end{array}\right]\left[\begin{array}{lll}
37 & 39.4 & 41
\end{array}\right]\left[\begin{array}{lll}
3.9917 & 4.1519 & 4.2587
\end{array}\right]\left[\begin{array}{llll}
0.5029 & 0.5433 & 0.5703
\end{array}\right]} \\
& {\left[\begin{array}{lll}
4.1264 & 4.7321 & 5.1358
\end{array}\right]\left[\begin{array}{lll}
29 & 32.6 & 35
\end{array}\right]\left[\begin{array}{llll}
3.6002 & 4.3466 & 4.8442
\end{array}\right]\left[\begin{array}{llll}
0.4190 & 0.4262 & 0.4310
\end{array}\right]} \\
& {\left[\begin{array}{lll}
3.6097 & 3.9617 & 4.1964
\end{array}\right]\left[\begin{array}{lll}
26 & 28.4 & 30
\end{array}\right]\left[\begin{array}{llll}
3.7239 & 4.1649 & 4.4590
\end{array}\right]\left[\begin{array}{llll}
0.5303 & 0.5474 & 0.5587
\end{array}\right]}
\end{aligned}
$$


The normalized three-parameter interval grey number evaluation matrix is

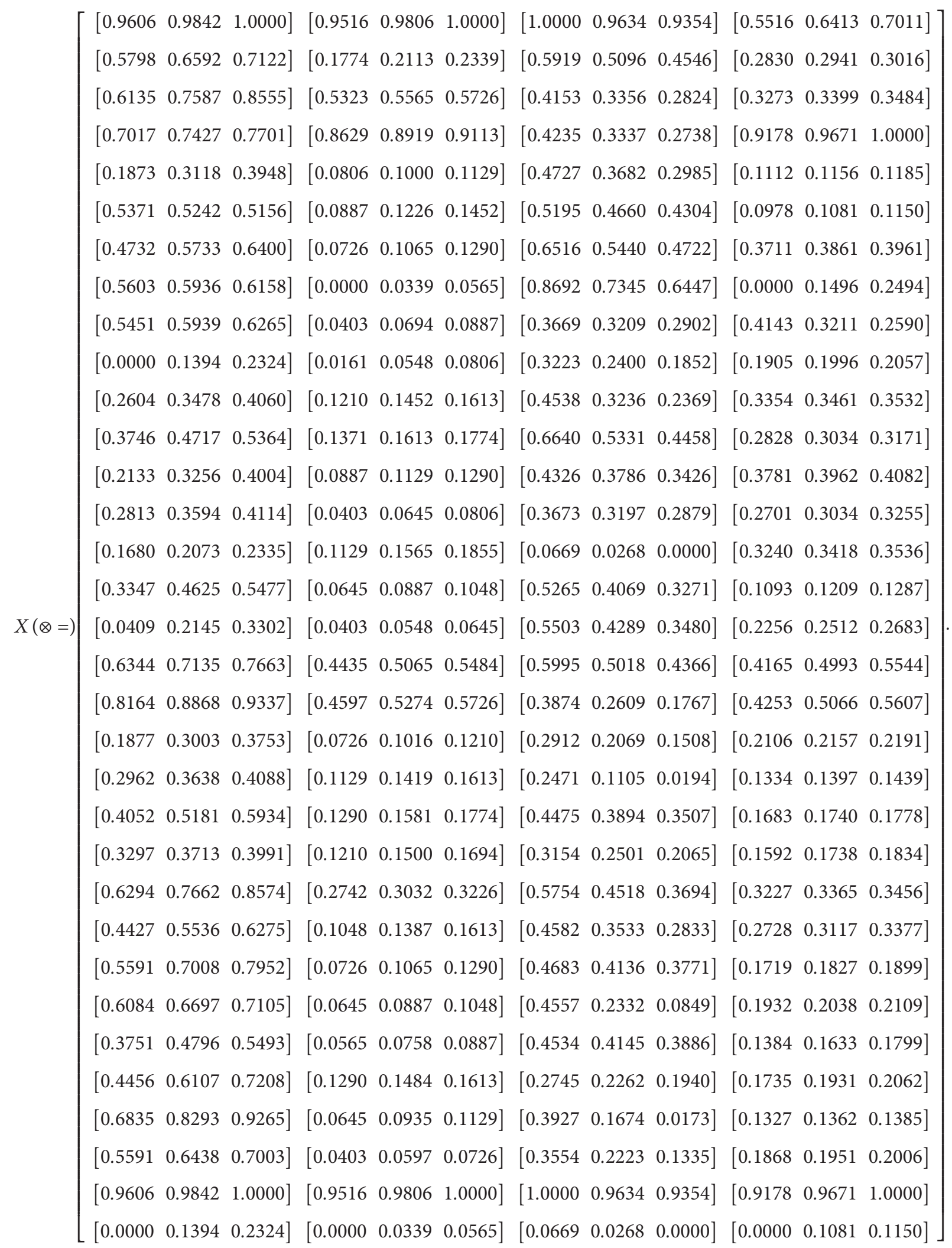


TABLE 7: $A-B$ comprehensive fuzzy judgment matrix.

\begin{tabular}{|c|c|c|c|c|c|c|c|c|c|c|c|c|}
\hline A & \multicolumn{3}{|c|}{$B 1$} & \multicolumn{3}{|c|}{$B 2$} & \multicolumn{3}{|c|}{$B 3$} & \multicolumn{3}{|c|}{$B 4$} \\
\hline$B 1$ & 0.5 & 0.5 & 0.5 & 0.292 & 0.342 & 0.392 & 0.517 & 0.567 & 0.617 & 0.463 & 0.513 & 0.563 \\
\hline$B 2$ & 0.611 & 0.661 & 0.711 & 0.5 & 0.5 & 0.5 & 0.563 & 0.613 & 0.663 & 0.544 & 0.594 & 0.644 \\
\hline B3 & 0.392 & 0.442 & 0.492 & 0.341 & 0.391 & 0.441 & 0.5 & 0.5 & 0.5 & 0.433 & 0.483 & 0.533 \\
\hline B4 & 0.442 & 0.492 & 0.542 & 0.365 & 0.415 & 0.465 & 0.471 & 0.521 & 0.571 & 0.5 & 0.5 & 0.5 \\
\hline
\end{tabular}

TABLe 8: Fuzzy complementary matrix.

\begin{tabular}{lcccc}
\hline & $B 1$ & $B 2$ & $B 3$ & $B 4$ \\
\hline$B 1$ & 0.500 & 0.342 & 0.567 & 0.513 \\
$B 2$ & 0.661 & 0.500 & 0.613 & 0.594 \\
$B 3$ & 0.442 & 0.391 & 0.500 & 0.483 \\
$B 4$ & 0.492 & 0.415 & 0.521 & 0.500 \\
\hline
\end{tabular}

TABle 9: Calculation results and comparison.

\begin{tabular}{|c|c|c|c|c|}
\hline Node & $G\left(x^{+}(\otimes), x_{i}(\otimes)\right)$ & $G\left(x^{-}(\otimes), x_{i}(\otimes)\right)$ & Linear correlation degree & Ranking of this article \\
\hline 1 & 0.7227 & 0.5026 & 0.6101 & 2 \\
\hline 2 & 0.6196 & 0.5950 & 0.5123 & 16 \\
\hline 3 & 0.6542 & 0.6244 & 0.5149 & 15 \\
\hline 4 & 0.8367 & 0.4228 & 0.7070 & 1 \\
\hline 5 & 0.6910 & 0.4836 & 0.6037 & 3 \\
\hline 6 & 0.5902 & 0.6887 & 0.4508 & 30 \\
\hline 7 & 0.7086 & 0.6146 & 0.5470 & 11 \\
\hline 8 & 0.5871 & 0.5846 & 0.5013 & 20 \\
\hline 9 & 0.5933 & 0.5873 & 0.5030 & 19 \\
\hline 10 & 0.6220 & 0.7173 & 0.4524 & 28 \\
\hline 11 & 0.7488 & 0.7116 & 0.5186 & 13 \\
\hline 12 & 0.6741 & 0.5362 & 0.5690 & 5 \\
\hline 13 & 0.6233 & 0.7190 & 0.4522 & 29 \\
\hline 14 & 0.8176 & 0.7023 & 0.5577 & 8 \\
\hline 15 & 0.8065 & 0.6980 & 0.5543 & 9 \\
\hline 16 & 0.7319 & 0.5711 & 0.5804 & 4 \\
\hline 17 & 0.7181 & 0.7309 & 0.4936 & 25 \\
\hline 18 & 0.6902 & 0.6997 & 0.4953 & 23 \\
\hline 19 & 0.6756 & 0.6864 & 0.4946 & 24 \\
\hline 20 & 0.7951 & 0.6931 & 0.5510 & 10 \\
\hline 21 & 0.6626 & 0.7156 & 0.4735 & 26 \\
\hline 22 & 0.6771 & 0.6590 & 0.5091 & 17 \\
\hline 23 & 0.6260 & 0.6117 & 0.5072 & 18 \\
\hline 24 & 0.6172 & 0.4806 & 0.5683 & 6 \\
\hline 25 & 0.6611 & 0.6591 & 0.5010 & 21 \\
\hline 26 & 0.6777 & 0.6429 & 0.5174 & 14 \\
\hline 27 & 0.6695 & 0.7229 & 0.4733 & 27 \\
\hline 28 & 0.6793 & 0.7852 & 0.4470 & 31 \\
\hline 29 & 0.6231 & 0.6245 & 0.4993 & 22 \\
\hline 30 & 0.6583 & 0.6132 & 0.5225 & 12 \\
\hline 31 & 0.6755 & 0.5465 & 0.5645 & 7 \\
\hline
\end{tabular}


TABLE 10: Ranking of node importance calculated in [17].

\begin{tabular}{|c|c|c|}
\hline Node & Correlation degree calculated in [17] & Ranking of [17] \\
\hline 1 & 0.6842 & 2 \\
\hline 2 & 0.5933 & 16 \\
\hline 3 & 0.5984 & 14 \\
\hline 4 & 0.6952 & 1 \\
\hline 5 & 0.6807 & 3 \\
\hline 6 & 0.5163 & 30 \\
\hline 7 & 0.6125 & 10 \\
\hline 8 & 0.5705 & 20 \\
\hline 9 & 0.5859 & 18 \\
\hline 10 & 0.5328 & 27 \\
\hline 11 & 0.6020 & 13 \\
\hline 12 & 0.6507 & 5 \\
\hline 13 & 0.5246 & 29 \\
\hline 14 & 0.6214 & 9 \\
\hline 15 & 0.6267 & 7 \\
\hline 16 & 0.6690 & 4 \\
\hline 17 & 0.5376 & 26 \\
\hline 18 & 0.5505 & 23 \\
\hline 19 & 0.5680 & 21 \\
\hline 20 & 0.6078 & 11 \\
\hline 21 & 0.5263 & 28 \\
\hline 22 & 0.5823 & 19 \\
\hline 23 & 0.5931 & 17 \\
\hline 24 & 0.6215 & 8 \\
\hline 25 & 0.5570 & 22 \\
\hline 26 & 0.5949 & 15 \\
\hline 27 & 0.5430 & 24 \\
\hline 28 & 0.5023 & 31 \\
\hline 29 & 0.5420 & 25 \\
\hline 30 & 0.6023 & 12 \\
\hline 31 & 0.6377 & 6 \\
\hline
\end{tabular}

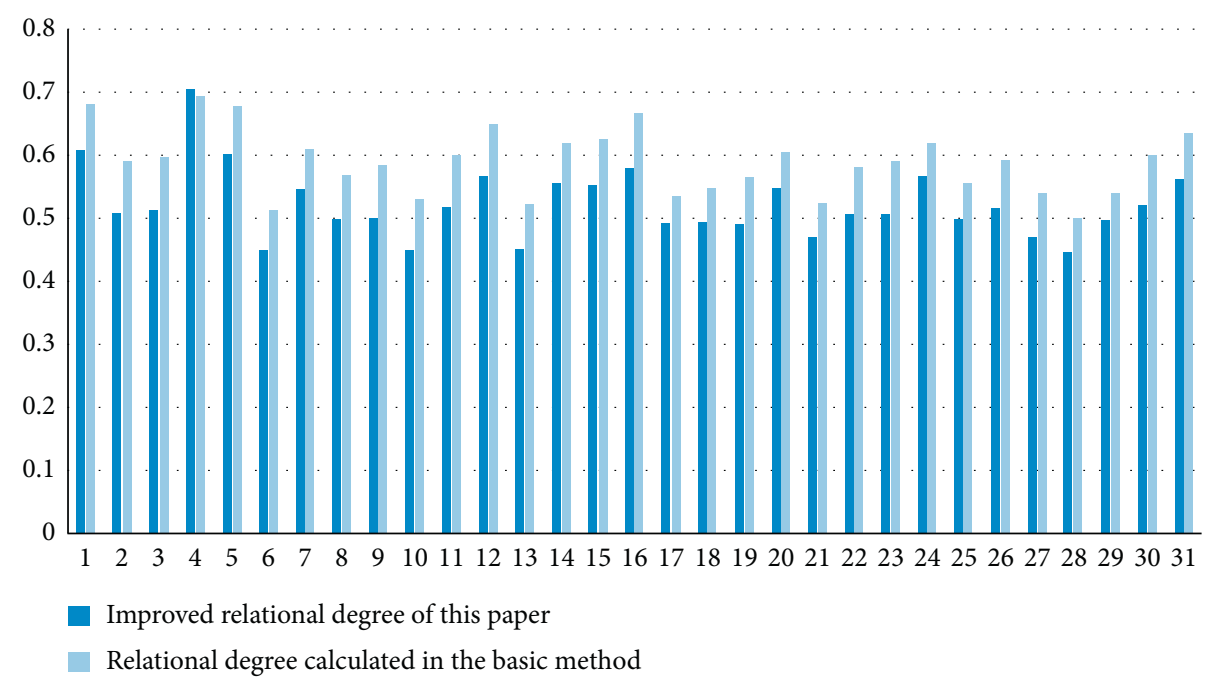

FIgURE 5: Comparison of relational degree results. 
According to formula (4), the effect evaluation vectors of ideal optimal scheme and critical scheme are obtained:

$$
\begin{aligned}
& X^{+}(\otimes)=\left(\left[\begin{array}{lllll}
0.9606 & 0.9842 & 1.0000
\end{array}\right],\left[\begin{array}{llll}
0.9516 & 0.9806 & 1.0000
\end{array}\right],\left[\begin{array}{llll}
1.0000 & 0.9634 & 0.9354
\end{array}\right],\left[\begin{array}{llll}
0.9178 & 0.9671 & 1.0000
\end{array}\right)\right. \\
& X^{-}(\otimes)=\left(\left[\begin{array}{llllll}
0.0000 & 0.1394 & 0.2324
\end{array}\right],\left[\begin{array}{lllll}
0.0000 & 0.0339 & 0.0565
\end{array}\right],\left[\begin{array}{llll}
0.0669 & 0.0268 & 0.0000
\end{array}\right],\left[\begin{array}{lll}
0.0000 & 0.1081 & 0.1150
\end{array}\right)\right.
\end{aligned}
$$

Then, we use TFAHP to calculate the weight:

The $A-B$ comprehensive fuzzy judgment matrix is obtained, as shown in Table 7.

The fuzzy complementary matrix is shown in Table 8 .

The relative weights of several indexes obtained by TFAHP are as follows:

$$
W=\left(w_{1}, w_{2}, w_{3}, w_{4}\right)=(0.19,0.32,0.21,0.28) .
$$

The weight obtained from the Gini coefficient is as follows: $W=\left(w_{1}, w_{2}, w_{3}, w_{4}\right)=(0.24,0.29,0.17,0.30)$.

Then, we can calculate the comprehensive weight $W=\left(w_{1}, w_{2}, w_{3}, w_{4}\right)=(0.235,0.30,0.18,0.285)$.

According to equations (8) and (10), the grey interval relational degree of each scheme with the ideal optimal scheme and the critical scheme can be obtained. Then, the linear relational degree is calculated by formula (5) (according to [28] and experts' opinion, $\lambda=0.5, \xi=0.5$, and $\left.\beta_{1}=\beta_{2}=0.5\right)$.

And, we also calculate the node importance ranking through the method of [17]. The above results are shown in Tables 9 and 10 and Figure 5.

According to the method of [17], the ranking of node importance is shown in Table 10.

Then, we compare the results of the two methods, as shown in Figure 5.

From the calculation results of this method and the comparison with other methods, we can see that the calculation results of the two methods are in the top several, respectively, impeller $>$ principal axis $>$ diffuser $>$ electrical machine $>$ evaporator $>$ evaporator $>$ condenser $>$ bend and impeller $>$ principal axis $>$ diffuser $>$ electrical machine $>$ refluxer $>$ condenser $>$ bearing $>$ evaporator. We can see that the ranking results of the two are roughly the same, except for some subtle differences. The ranking results are realistic and roughly the same. However, we can see from the graphic difference in Figure 5 that the ranking difference obtained by the method proposed in this paper is stronger. This method makes the ranking more obvious and easy to identify. The evaluation method proposed in this paper increases the operability and identifiability of the importance ranking of design elements in the design process.

\section{Conclusion}

In order to comprehensively describe the complex product design process, this paper uses the complex product FBS model to describe the complex detailed design relationship. Then, the identification index system of important parts in the complex product design stage is constructed according to the characteristics of network. The three-parameter interval grey number relational model is improved based on the fuzzy analytic hierarchy process. The comparison of experimental results shows that the results calculated by the proposed method are more representative. It also shows that the method is practical and effective.

The multilevel description model of the design process based on FBS proposed in this paper can describe the design process in more detail, and the analysis of the detailed design process is more specific. The evaluation of important parts based on this is more scientific. In addition, the weight improvement of the three-parameter interval grey number based on the fuzzy analytic hierarchy process and the Gini coefficient method is in line with the law of actual data and is more effective. The research comparison of this paper also shows the effectiveness and feasibility of the proposed method.

The next research prospect of this paper is to further improve the objectivity of data acquisition in the design stage and reduce the proportion of experience data of designers. Subsequently, we will try to establish a more scientific design database, standardize the management of its design process, and make its data acquisition more accurate and scientific. It will provide service structure for each department to obtain information, which makes the research more practical.

\section{Data Availability}

All data generated and materials or analyzed during this study are included in this published article.

\section{Ethical Approval}

This article does not involve the content of violating ethics and morality.

\section{Conflicts of Interest}

The authors declare that they have no conflicts of interest.

\section{Authors' Contributions}

Weiming Yang, Congdong Li, and Yinyun Yu conceptualized the study; Weiming Yang and Congdong Li proposed the model; Weiming Yang and Mingsheng Zhong carried out the case study; Weiming Yang, Congdong Li, Yinyun Yu, and Mingsheng Zhong wrote, reviewed, and edited the manuscript. All authors have read and agreed to participate and publish the manuscript. 


\section{Acknowledgments}

This work was supported by the National Natural Science Foundation of China (no. 72072072), National Natural Science Foundation of China (no. 71672074), Natural Science Foundation of Guangdong Province of China (no. 2019A1515010045), and 2018 Guangzhou Leading Innovation Team Program, China (no. 201909010006).

\section{References}

[1] L. Luo, J. Wang, and K. Zhang, "A model for complex product development performance based on knowledge management," Operations Research and Management Science, vol. 26, no. 1, pp. 166-172, 2017.

[2] R. Magnaye, B. Sauser, P. Patanakul, D. Nowicki, and W. Randall, "Earned readiness management for scheduling, monitoring and evaluating the development of complex product systems," International Journal of Project Management, vol. 32, no. 7, pp. 1246-1259, 2014.

[3] C.-Y. Huang, C.-L. Lee, T.-H. Wen, and C.-T. Sun, "A computer virus spreading model based on resource limitations and interaction costs," Journal of Systems and Software, vol. 86, no. 3, pp. 801-808, 2013.

[4] M. Jalili and M. Perc, "Information cascades in complex networks," Journal of Complex Networks, vol. 5, no. 5, pp. 665-693, 2017.

[5] C. Nowzari, V. M. Preciado, and G. J. Pappas, “Analysis and control of epidemics: a survey of spreading processes on complex networks," IEEE Control Systems, vol. 36, no. 1, pp. 26-46, 2016.

[6] F. Lu, W. Zhang, L. Shao, X. Jiang, P. Xu, and H. Jin, "Scalable influence maximization under independent cascade model," Journal of Network and Computer Applications, vol. 86, pp. 15-23, 2017.

[7] M. Gong, J. Yan, B. Shen, L. Ma, and Q. Cai, "Influence maximization in social networks based on discrete particle swarm optimization," Information Sciences, vol. 367-368, pp. 600-614, 2016.

[8] Z.-K. Bao, J.-G. Liu, and H.-F. Zhang, "Identifying multiple influential spreaders by a heuristic clustering algorithm," Physics Letters A, vol. 381, no. 11, pp. 976-983, 2017.

[9] L. Z. Cui, H. X. Hu, S. Yu et al., "DDSE: a novel evolutionary algorithm based on degree-descending search strategy for influence maximization in social networks," Journal of Network and Computer Applications, vol. 103, pp. 976-983, 2018.

[10] H. Li, R. Zhang, Z. Zhao, and X. Liu, "LPA-MNI: an improved label propagation algorithm based on modularity and node importance for community detection," Entropy, vol. 23, no. 5, p. 497, 2021.

[11] Z. N. Berberler, H. I. Yildirim, T. Iltuzer, and I. Tunc, “Agglomeration-based node importance analysis in wheel-type networks," International Journal of Foundations of Computer Science, vol. 32, no. 3, pp. 269-288, 2021.

[12] Q. Sun, G. Yang, and A. Zhou, "An entropy-based selfadaptive node importance evaluation method for complex networks," Complexity, vol. 2020, Article ID 4529429, 13 pages, 2020.

[13] F. Bencherif and L. H. Mouss, "Complex network to enhance characterization analysis in modelling product development process," African Journal of Science, Technology, Innovation and Development, vol. 12, no. 7, pp. 797-811, 2020.

[14] R. Li, N. Yang, Y. Zhang, H. Liu, and M. Zhang, "Impacts of module-module aligned patterns on risk cascading propagation in complex product development (CPD) interdependent networks," Physica A-Statistical Mechanics and Its Applications, vol. 564, 2021.

[15] R. Li, H. Yi, and H. Cao, "Towards understanding dynamic design change propagation in complex product development via complex network approach," International Journal of Production Research, vol. 18, 2021.

[16] T. Li, H. Chen, J. Yuan, J. Qian, and A. W. Siyal, "Quality risk propagation of complex product collaborative manufacturing supply chain network based on CN and SoV," Discrete Dynamics in Nature and Society, vol. 2020, Article ID 8889903, 16 pages, 2020.

[17] X. Yin, Y. Mo, C. Dong, and Y. Zhang, "Identification of the influential parts in a complex mechanical product from a reliability perspective using complex network theory," Quality and Reliability Engineering International, vol. 36, no. 2, pp. 604-622, 2020.

[18] G. Yu, Y. Yang, X. Zhang, and C. Li, "Network-based analysis of requirement change in customized complex product development," International Journal of Information Technology and Decision Making, vol. 36, pp. 604-622, 2020.

[19] D. Zhang, Z. Zhao, Y. Zhou, and Y. Guo, "A novel complex network-based modeling method for heterogeneous product design," Cluster Computing-the Journal of Networks Software Tools and Applications, vol. 22, pp. S7861-S7872, 2019.

[20] N. Zhang, M. Li, H. Ren, and Y. Li, "A network-based fourphase routing approach of multisource design change propagation on complex products," Kybernetes, 2020, in press.

[21] N. Zhang, Y. Yang, Y. Zheng, and J. Su, "Module partition of complex mechanical products based on weighted complex networks," Journal of Intelligent Manufacturing, vol. 30, no. 4, pp. 1973-1998, 2019.

[22] Y. Li and D. Zhang, "Multi-attribute group grey target decision-making method based on three-parameter interval grey number," Journal of Grey System, vol. 32, pp. 96-109, 2020.

[23] M. Li, K. Chen, C. Liu, and B. Liu, "The interval parameter optimization model based on three-way decision space and its application on "green products recommendation," Discrete Dynamics in Nature and Society, vol. 2020, 2020.

[24] K. Chen and P. Chen, "Decision making method of TOPSIS based on three-parameter interval grey numbers," Systems Engineering and Electronics, vol. 41, pp. 124-130, 2019.

[25] Y. Li and D. Zhang, "Dynamic multi-attribute decisionmaking method with three-parameter interval grey number based on the prospect theory," Grey Systems: Theory and Application, vol. 8, no. 4, pp. 424-435, 2018.

[26] Y. Li, Y. Niu, W. Wang, and B. Li, "Grey-incidence Clustering Decision-making method with Three-parameter interval grey number based on Regret theory," in Proceedimgs of the 2017 International Conference on Grey Systems and Intelligent Services (GSIS), IEEE, Stockholm, Sweden, August 2017.

[27] C. Li and J. Yuan, "A new multi-attribute decision-making method with three-parameter interval grey linguistic variable," International Journal of Fuzzy Systems, vol. 19, no. 2, pp. 292-300, 2017.

[28] X. Wang and Y. G. Dang, "Dynamic multiattribute decisionmaking methods with three-parameter interval grey number," Control and Decision, vol. 30, no. 09, pp. 1623-1629, 2015.

[29] D. Luo, "Decision-making method with three-parameter interval grey number," System Engineering-Theory \& Practice, vol. 29, no. 01, pp. 124-130, 2009.

[30] S. L. Yan, S. F. Liu, J. J. Zhu, Z. G. Fang, and J. Liu, "TOPSIS decision-making method with three-parameter interval number based on entropy measure," Chinese Journal of Management Science, vol. 21, pp. 145-151, 2013. 
[31] J. Y. Dong, S. P. Wan, and S. M. Chen, "Fuzzy best-worst method based on triangular fuzzy numbers for multi-criteria decision-making," Information Sciences, vol. 547, no. 08, pp. 1080-1104, 2021.

[32] S. P. Wan, Z. H. Chen, and J. Y. Dong, "An integrated interval type-2 fuzzy technique for democratic-autocratic multi-criteria decision making," Knowledge-Based Systems, vol. 214, Article ID 106735, 2021.

[33] S. P. Wan and J. Y. Dong, "A novel extension of best-worst method with intuitionistic fuzzy reference comparisons," IEEE Transactions on Fuzzy Systems, p. 1, 2021, https:// ieeexplore.ieee.org/document/9373994.

[34] S. P. Wan, J. Y. Dong, and S. M. Chen, "Fuzzy best-worst method based on generalized interval-valued trapezoidal fuzzy numbers for multi-criteria decision-making," Information Sciences, vol. 573, pp. 493-518, 2021.

[35] M. H. Lu and K. P. Zhu, "Improved analytic hierarchy process based on triangular fuzzy number," in Proceedings of the 2nd International Conference on Education, Economics and Management Research (ICEEMR), Singapore, June 2018.

[36] L. Jiang, T. Tao, C. Zhang, H. Jiang, and J. Wang, "Summary of the port shoreline resource evaluation based on triangular fuzzy analytic hierarchy process," Polish Maritime Research, vol. 24, no. s3, pp. 16-22, 2017.

[37] J. Correa-Parra, J. Francisco Vergara-Perucich, and C. Aguirre-Nunez, "Water privatization and inequality: Gini coefficient for water resources in Chile," Water, vol. 12, no. 12, Article ID 3369, 2020. 